\title{
Nonlinear Instability for a Leslie-Gower Predator-Prey Model with Cross Diffusion
}

\author{
Lina Zhang and Shengmao Fu \\ Department of Mathematics, Northwest Normal University, Lanzhou 730070, China \\ Correspondence should be addressed to Shengmao Fu; fusm@nwnu.edu.cn
}

Received 18 July 2013; Accepted 29 August 2013

Academic Editor: Benchawan Wiwatanapataphee

Copyright (C) 2013 L. Zhang and S. Fu. This is an open access article distributed under the Creative Commons Attribution License, which permits unrestricted use, distribution, and reproduction in any medium, provided the original work is properly cited.

A rigorous mathematical characterization for early-stage spatial and temporal patterns formation in a Leslie-Gower predator-prey model with cross diffusion is investigated. Given any general perturbation near an unstable constant equilibrium, we prove that its nonlinear evolution is dominated by the corresponding linear dynamics along a fixed finite number of the fastest growing modes.

\section{Introduction}

Since Turing proposed the striking idea of "diffusion-driven instability" in 1952 [1], reaction-diffusion systems are often employed to investigate chemical and biological pattern formations and have received much attention from the scientists [2-7]. However, most of the works concentrate on pattern formation in the case of linear instability, and there is a little discussion about the nonlinear effect of a reaction-diffusion system on the evolution of a nonuniform pattern.

In general, nonlinear instability is treated with great delicacy and difficulty. At first, nonlinear instability was established for nondissipative systems [8-11]. In 2004, Guo et al. [12] established nonlinear instability for an unstable Kirchhoff ellipse. Based upon a precise linear analysis, they found that the dynamics of general perturbation can be characterized by the linear dynamics of the fastest growing modes. This marks a beginning of a quantitative description of instability. Subsequently, Guo and Hwang dealt with nonlinear stability for a Keller-Segel model in [13] and described the early-stage pattern formation in that model.

Recently, Guo and Hwang considered the following reaction-diffusion system [14]

$$
\begin{aligned}
& \frac{\partial U}{\partial t}=\nabla \cdot\left(D_{1}(U, V) \nabla U\right)+f(U, V), \\
& \frac{\partial V}{\partial t}=\nabla \cdot\left(D_{2}(U, V) \nabla V\right)+g(U, V),
\end{aligned}
$$

in a box $\mathbb{T}^{N}=(0, \pi)^{N} \subset \mathbb{R}^{N}(N \leq 3)$ with the homogeneous Neumann boundary conditions. In system $(1), U(x, t), V(x, t)$ denote the densities of two interactive species at time $t$, the functions $D_{1}, D_{2}$ are their diffusion rates, and $f, g$ are the reaction functions. The classical Turing instability and Turing patterns were studied under some suitable conditions. Their result shows that the nonlinear evolution of patterns is dominated by the corresponding linear dynamics along a fixed finite number of the fastest growing modes over a time period.

In this paper, we consider the following Leslie-Gower predator-prey model with cross diffusion:

$$
\begin{gathered}
u_{t}-d_{1} \Delta u=\lambda u-u^{2}-\beta u v, \quad x \in \mathbb{T}^{N}, t>0, \\
v_{t}-d_{2} \Delta\left[\left(1+d_{3} u\right) v\right]=v\left(\mu-\frac{v}{m+u}\right), \quad x \in \mathbb{T}^{N}, t>0, \\
\frac{\partial u}{\partial x_{i}}=\frac{\partial v}{\partial x_{i}}=0, \quad x_{i}=0, \pi, i=1, \ldots, N, t>0, \\
u(x, 0)=u_{0}(x), \quad v(x, 0)=v_{0}(x), \quad x \in \mathbb{T}^{N},
\end{gathered}
$$

where $u(x, t)$ and $v(x, t)$ represent the densities of the species prey and predator, respectively. The parameters $\lambda, \beta, \mu, m, d_{1}$, $d_{2}$, and $d_{3}$ are all positive constants, where $\lambda$ and $\mu$ are the intrinsic growth rates of the prey and predator, $\beta$ is the predation rate, and the term $v /(m+u)$ is a modified Leslie-Gower term [15]. The constants $d_{1}, d_{2}$, called diffusion 
coefficients, represent the natural tendency of each species to diffuse to areas of smaller population concentration, and $d_{3}$, called a cross-diffusion coefficient, expresses the population flux of the predator resulting from the presence of the prey species. For more ecological backgrounds about this model, one can refer to [15-17].

System (2) and its variants were studied widely for pattern formation by applying the bifurcation theory and the degree theory $[6,18-20]$ in the case of linear instability. Inspired by the works $[13,14]$, in this paper, we attempt to study the nonlinear instability for this system and give a rigorous mathematical characterization for the nonlinear evolution of pattern by using a bootstrap technique. The mathematical approach in this paper is similar in spirit to that of $[13,14]$. However, our problem (2) is much more complex. Notice that the diffusion term of the predator equation in the model (2) is

$$
d_{2} \Delta\left[\left(1+d_{3} u\right) v\right]=\nabla \cdot\left[d_{2} d_{3} v \nabla u+d_{2}\left(1+d_{3} u\right) \nabla v\right]
$$

In some sense, the coupled degree in (2) is stronger than that in (1). As a result, our analysis here, especially in establishing $H^{2}$ estimates for nonlinear terms $d_{2} d_{3} \nabla(v \nabla u)$ and $d_{2} \nabla\left[\left(1+d_{3} u\right) \nabla v\right]$, is much more difficult and requires some techniques beyond those of $[13,14]$.

It is obvious that (2) has a unique positive equilibrium $(\widetilde{u}, \widetilde{v})$ if and only if $\lambda>\beta \mu m$, where

$$
\tilde{u}=\frac{\lambda-\beta \mu m}{1+\beta \mu}, \quad \widetilde{v}=\frac{\mu(m+\lambda)}{1+\beta \mu} .
$$

Let $\widehat{u}=u(x, t)-\widetilde{u}, \widehat{v}=v(x, t)-\widetilde{v}$ be the perturbation around $(\widetilde{u}, \widetilde{v})$ and still denote it by $(u, v)$. Then, the perturbation $(u, v)$ satisfies the following strongly coupled equations:

$$
\begin{gathered}
u_{t}-d_{1} \Delta u=g_{1}(u+\widetilde{u}, v+\widetilde{v}), \quad x \in \mathbb{T}^{N}, t>0, \\
v_{t}-d_{2} \Delta\left[\left(1+d_{3}(u+\widetilde{u})\right)(v+\widetilde{v})\right] \\
=g_{2}(u+\widetilde{u}, v+\widetilde{v}), \quad x \in \mathbb{T}^{N}, t>0, \\
\frac{\partial u}{\partial x_{i}}=\frac{\partial v}{\partial x_{i}}=0, \quad x_{i}=0, \pi, i=1, \ldots, N, t>0, \\
u(x, 0)=u_{0}(x), \quad v(x, 0)=v_{0}(x), \quad x \in \mathbb{T}^{N},
\end{gathered}
$$

where

$$
g_{1}(u, v)=\lambda u-u^{2}-\beta u v, \quad g_{2}(u, v)=v\left(\mu-\frac{v}{m+u}\right) \text {. }
$$

This paper is organized as follows. In Section 2, the growing modes in the linearized system are studied, which are important for our later discussions. Section 3 gives some estimates for the perturbation. The key is to control the nonlinear growth of high-order energy. In Section 4, the nonlinear instability is obtained.

\section{Growing Modes in the Linearized System}

The corresponding linearized system of (5) takes the form of

$$
\begin{gathered}
u_{t}-d_{1} \Delta u=-\tilde{u} u-\beta \tilde{u} v, \\
v_{t}-d_{2} d_{3} \widetilde{v} \Delta u-d_{2}\left(1+d_{3} \tilde{u}\right) \Delta v=\mu^{2} u-\mu v .
\end{gathered}
$$

We use $[\cdot, \cdot]$ to denote a column vector and let $\mathbf{w}(x, t)=$ $[u(x, t), v(x, t)], \mathbf{q}=\left(q_{1}, \ldots, q_{N}\right) \in \mathbb{N}^{N}$. Then, $\mathbf{q}^{2}=$ $\sum_{i=1}^{N} q_{i}^{2}$ are eigenvalues of $-\Delta$ on $\mathbb{T}^{N}$ under the homogeneous Neumann boundary condition, and the corresponding normalized eigenfunctions are given by

$$
e_{\mathbf{q}}(x)= \begin{cases}\left(\frac{1}{\pi}\right)^{N / 2}, & \mathbf{q}=\mathbf{0}, \\ \left(\frac{2}{\pi}\right)^{N / 2} \prod_{i=1}^{N} \cos \left(q_{i} x_{i}\right), & \mathbf{q} \neq \mathbf{0} .\end{cases}
$$

This set of eigenfunctions forms an orthonormal basis in $L^{2}\left(\mathbb{T}^{N}\right)$.

We look for a normal mode to be the linear system (7) of the following form:

$$
\mathbf{w}(x, t)=\mathbf{r}_{\mathbf{q}} e^{\lambda_{\mathbf{q}} t} e_{\mathbf{q}}(x),
$$

where $\lambda_{\mathbf{q}}$ is a complex number and $\mathbf{r}_{\mathbf{q}}$ is a vector; they depend on q. Substituting (9) into (7), we have

$$
\lambda_{\mathbf{q}} \mathbf{r}_{\mathbf{q}}=\left(\begin{array}{cc}
-\tilde{u}-d_{1} \mathbf{q}^{2} & -\beta \tilde{u} \\
\mu^{2}-d_{2} d_{3} \widetilde{v} \mathbf{q}^{2} & -\mu-d_{2}\left(1+d_{3} \tilde{u}\right) \mathbf{q}^{2}
\end{array}\right) \mathbf{r}_{\mathbf{q}}:=L \mathbf{r}_{\mathbf{q}} .
$$

System (7) possesses a nontrivial normal mode if and only if

$$
\operatorname{det}\left(\begin{array}{cc}
\lambda_{\mathbf{q}}+\tilde{u}+d_{1} \mathbf{q}^{2} & \beta \tilde{u} \\
-\mu^{2}+d_{2} d_{3} \widetilde{v} \mathbf{q}^{2} & \lambda_{\mathbf{q}}+\mu+d_{2}\left(1+d_{3} \tilde{u}\right) \mathbf{q}^{2}
\end{array}\right)=0,
$$

which is equivalent to

$$
\begin{aligned}
\lambda_{\mathbf{q}}^{2} & +\left\{\tilde{u}+\mu+\left[d_{1}+d_{2}\left(1+d_{3} \tilde{u}\right)\right] \mathbf{q}^{2}\right\} \lambda_{\mathbf{q}}+d_{1} d_{2}\left(1+d_{3} \widetilde{u}\right) \mathbf{q}^{4} \\
& +\left[d_{1} \mu+d_{2}\left(1+d_{3} \widetilde{u}\right) \tilde{u}-\beta d_{2} d_{3} \widetilde{u} \widetilde{v}\right] \mathbf{q}^{2}+\mu \widetilde{u}+\beta \mu^{2} \widetilde{u}=0
\end{aligned}
$$

Thus, we deduce the following well-known aggregation (i.e., linear instability) criterion by requiring that there exists a $\mathbf{q}$, such that the constant term in (12) is

$$
\begin{aligned}
& d_{1} d_{2}\left(1+d_{3} \tilde{u}\right) \mathbf{q}^{4}+\left[d_{1} \mu+d_{2}\left(1+d_{3} \tilde{u}\right) \tilde{u}-\beta d_{2} d_{3} \tilde{u} \widetilde{v}\right] \mathbf{q}^{2} \\
& +\mu \tilde{u}+\beta \mu^{2} \tilde{u}<0 .
\end{aligned}
$$

In this paper, we always assume that there exists a $\mathbf{q}$, such that (13) holds. Then, the discriminant of (12) is

$$
\begin{aligned}
\Delta= & {\left[d_{1}-d_{2}\left(1+d_{3} \tilde{u}\right)\right]^{2} \mathbf{q}^{4} } \\
+ & \left\{2(\tilde{u}+\mu)\left[d_{1}+d_{2}\left(1+d_{3} \tilde{u}\right)\right]\right. \\
& \left.\quad-4\left[d_{1} \mu+d_{2}\left(1+d_{3} \tilde{u}\right) \tilde{u}\right]+4 \beta d_{2} d_{3} \tilde{u} \widetilde{v}\right\} \mathbf{q}^{2} \\
& +(\tilde{u}-\mu)^{2}-4 \beta \mu^{2} \tilde{u},
\end{aligned}
$$


and the coefficient of $\mathbf{q}^{2}$ is positive, since (13) implies

$$
d_{1} \mu+d_{2}\left(1+d_{3} \tilde{u}\right) \tilde{u}-\beta d_{2} d_{3} \tilde{u} \tilde{v}<0 .
$$

For given $\mathbf{q}$, we denote the corresponding eigenvalues by $\lambda_{\mathbf{q}}^{ \pm}$and eigenvectors by $\mathbf{r}_{ \pm}(\mathbf{q})$. We split it into three cases for the linear analysis.

(1) $\Delta>0$. Let $\Lambda_{1}=\{\mathbf{q} \mid \Delta>0\}$, and let $\lambda_{\mathbf{q}}^{ \pm}$be two distinct real roots with $\lambda_{\mathbf{q}}^{+}>\lambda_{\mathbf{q}}^{-}, \lambda_{\mathbf{q}}^{ \pm}$being the corresponding (linearly independent) real eigenvectors. It is easy to see that

$$
\mathbf{r}_{ \pm}(\mathbf{q})=\left[1,-\frac{\lambda_{\mathbf{q}}^{ \pm}+\tilde{u}+d_{1} \mathbf{q}^{2}}{\beta \tilde{u}}\right] .
$$

Denote

$$
\begin{aligned}
\Lambda_{*}= & \left\{\mathbf{q} \mid d_{1} d_{2}\left(1+d_{3} \tilde{u}\right) \mathbf{q}^{4}\right. \\
& +\left[d_{1} \mu+d_{2}\left(1+d_{3} \tilde{u}\right) \tilde{u}-\beta d_{2} d_{3} \tilde{u} \widetilde{v}\right] \mathbf{q}^{2} \\
& \left.+\mu \tilde{u}+\beta \mu^{2} \tilde{u}<0\right\} .
\end{aligned}
$$

Clearly, $\lambda_{\mathbf{q}}^{+}>0$ for $\mathbf{q} \in \Lambda_{*}$. Note that there are only finitely many $\mathbf{q}$ in $\Lambda_{*}$ and $\Lambda_{*} \subset \Lambda_{1}$. Therefore, there are only finitely many linear growing modes, such that the constant equilibrium $(0,0)$ of $(5)$ is unstable. Furthermore, we define

$$
\lambda_{\max }=\max _{\lambda_{\mathbf{q}}^{+}>0} \lambda_{\mathbf{q}}^{+}, \quad \Lambda_{\max }=\left\{\mathbf{q} \mid \lambda_{\mathbf{q}}^{+}=\lambda_{\max }\right\} .
$$

Then, $\Lambda_{\max } \subset \Lambda_{*} \subset \Lambda_{1}$.

(2) $\Delta=0$. Let $\Lambda_{2}=\{\mathbf{q} \mid \Delta=0\}$. In this case, (12) possesses repeated real eigenvalues. Consider

$\lambda_{\mathbf{q}}=\lambda_{\mathbf{q}}^{+}=\lambda_{\mathbf{q}}^{-}=-\frac{1}{2}\left\{\tilde{u}+\mu+\left[d_{1}+d_{2}\left(1+d_{3} \tilde{u}\right)\right] \mathbf{q}^{2}\right\}<0$.

The corresponding eigenvectors are

$$
\mathbf{r}(\mathbf{q})=\mathbf{r}_{+}(\mathbf{q})=\mathbf{r}_{-}(\mathbf{q})=\left[1,-\frac{\lambda_{\mathbf{q}}+\tilde{u}+d_{1} \mathbf{q}^{2}}{\beta \tilde{u}}\right],
$$

and we can find another independent vector $\mathbf{r}^{\prime}(\mathbf{q})=$ $[0,-1 /(\beta \widetilde{u})]$, satisfying

$$
\left(L-\lambda_{\mathbf{q}} I\right) \mathbf{r}^{\prime}(\mathbf{q})=\mathbf{r}(\mathbf{q}) .
$$

(3) $\Delta<0$. The complex case is where (12) possesses a pair of complex eigenvalues with a negative real part. Denote $\Lambda_{3}=\{\mathbf{q} \Delta<0\}$, and for any $\mathbf{q} \in \Lambda_{3}$, denote

$$
\lambda_{\mathbf{q}}^{+}=\operatorname{Re} \lambda_{\mathbf{q}}+i \operatorname{Im} \lambda_{\mathbf{q}}, \quad \mathbf{r}_{+}(\mathbf{q})=\operatorname{Re} \mathbf{r}(\mathbf{q})+i \operatorname{Im} \mathbf{r}(\mathbf{q}) .
$$

Then,

$$
\lambda_{\mathbf{q}}^{-}=\operatorname{Re} \lambda_{\mathbf{q}}-i \operatorname{Im} \lambda_{\mathbf{q}}, \quad \mathbf{r}_{-}(\mathbf{q})=\operatorname{Re} \mathbf{r}(\mathbf{q})-i \operatorname{Im} \mathbf{r}(\mathbf{q}),
$$

where $\operatorname{Re} \mathbf{r}(\mathbf{q})$ and $\operatorname{Im} \mathbf{r}(\mathbf{q})$ are linearly independent vectors.
Given any initial perturbation $\mathbf{w}(x, 0)$, we can expand it as follows:

$$
\begin{aligned}
\mathbf{w}(x, 0)= & \sum_{\mathbf{q} \in \Lambda_{1}}\left\{w_{\mathbf{q}}^{-} \mathbf{r}_{-}(\mathbf{q})+w_{\mathbf{q}}^{+} \mathbf{r}_{+}(\mathbf{q})\right\} e_{\mathbf{q}}(x) \\
& +\sum_{\mathbf{q} \in \Lambda_{2}}\left\{w_{\mathbf{q}} \mathbf{r}(\mathbf{q})+w_{\mathbf{q}}^{\prime} \mathbf{r}^{\prime}(\mathbf{q})\right\} e_{\mathbf{q}}(x) \\
& +\sum_{\mathbf{q} \in \Lambda_{3}}\left\{w_{\mathbf{q}}^{\operatorname{Re}} \operatorname{Re} \mathbf{r}(\mathbf{q})+w_{\mathbf{q}}^{\operatorname{Im}} \operatorname{Im} \mathbf{r}(\mathbf{q})\right\} e_{\mathbf{q}}(x) \\
:= & \sum_{\mathbf{q} \in \mathbb{N}^{N}} \mathbf{w}_{\mathbf{q}} e_{\mathbf{q}}(x),
\end{aligned}
$$

where $w_{\mathbf{q}}^{-}, w_{\mathbf{q}}^{+}, w_{\mathbf{q}}, w_{\mathbf{q}}^{\prime}, w_{\mathbf{q}}^{\mathrm{Re}}$, and $w_{\mathbf{q}}^{\mathrm{Im}}$ are constants, and

$$
\begin{gathered}
\mathbf{w}_{\mathbf{q}}=w_{\mathbf{q}}^{-} \mathbf{r}_{-}(\mathbf{q})+w_{\mathbf{q}}^{+} \mathbf{r}_{+}(\mathbf{q}), \quad \mathbf{q} \in \Lambda_{1}, \\
\mathbf{w}_{\mathbf{q}}=w_{\mathbf{q}} \mathbf{r}(\mathbf{q})+w_{\mathbf{q}}^{\prime} \mathbf{r}^{\prime}(\mathbf{q}), \quad \mathbf{q} \in \Lambda_{2}, \\
\mathbf{w}_{\mathbf{q}}=w_{\mathbf{q}}^{\operatorname{Re}} \operatorname{Re} \mathbf{r}(\mathbf{q})+w_{\mathbf{q}}^{\operatorname{Im}} \operatorname{Im} \mathbf{r}(\mathbf{q}), \quad \mathbf{q} \in \Lambda_{3} .
\end{gathered}
$$

The unique solution $\mathbf{w}(x, t)=[u(x, t), v(x, t)]$ to $(7)$ is given by

$$
\begin{aligned}
& \mathbf{w}(x, t)=\sum_{\mathbf{q} \in \Lambda_{1}}\left\{w_{\mathbf{q}}^{-} \mathbf{r}_{-}(\mathbf{q}) e^{\lambda_{\mathbf{q}}^{-} t}+w_{\mathbf{q}}^{+} \mathbf{r}_{+}(\mathbf{q}) e^{\lambda_{\mathbf{q}}^{+} t}\right\} e_{\mathbf{q}}(x) \\
& +\sum_{\mathbf{q} \in \Lambda_{2}}\left\{w_{\mathbf{q}} \mathbf{r}(\mathbf{q})+w_{\mathbf{q}}^{\prime} \mathbf{r}^{\prime}(\mathbf{q})+w_{\mathbf{q}}^{\prime} \mathbf{r}(\mathbf{q}) t\right\} e^{\lambda_{\mathbf{q}} t} e_{\mathbf{q}}(x) \\
& +\sum_{\mathbf{q} \in \Lambda_{3}}\left\{w _ { \mathbf { q } } ^ { \operatorname { R e } } \left(\operatorname{Re} \mathbf{r}(\mathbf{q}) \cos \left[\left(\operatorname{Im} \lambda_{\mathbf{q}}\right) t\right]\right.\right. \\
& \left.-\operatorname{Im} \mathbf{r}(\mathbf{q}) \sin \left[\left(\operatorname{Im} \lambda_{\mathbf{q}}\right) t\right]\right) \\
& +w_{\mathbf{q}}^{\operatorname{Im}}\left(\operatorname{Re} \mathbf{r}(\mathbf{q}) \sin \left[\left(\operatorname{Im} \lambda_{\mathbf{q}}\right) t\right]\right. \\
& \left.\left.+\operatorname{Im} \mathbf{r}(\mathbf{q}) \cos \left[\left(\operatorname{Im} \lambda_{\mathbf{q}}\right) t\right]\right)\right\} \\
& \times e^{\left(\operatorname{Re} \lambda_{\mathbf{q}}\right) t} e_{\mathbf{q}}(x) \\
& :=e^{L t} \mathbf{w}(x, 0) .
\end{aligned}
$$

In the sequel, the constant $C_{0}$ will only depend on the domain $\mathbb{T}^{N}$ and the dimension $N$, and the generic constants $C_{1}, C_{2}, \widehat{C}_{1}, \widehat{C}_{2}$, and so forth will depend on $\mathbb{T}^{N}, N$, and the parameters $\lambda, \beta, m, \mu, d_{1}, d_{2}$, and $d_{3}$. Our main result of this section is the following lemma.

Lemma 1. Assume that the instability criterion (13) is valid. Suppose that

$$
\mathbf{w}(x, t)=[u(x, t), v(x, t)]:=e^{L t} \mathbf{w}(x, 0)
$$

is a solution to the linearized system (7) with the initial condition $\mathbf{w}(x, 0)$. Then, there exists a constant $\widehat{C}_{1} \geq 1$, such that

$$
\|\mathbf{w}(\cdot, t)\|_{L^{2}\left(\mathbb{T}^{N}\right)} \leq \widehat{C}_{1} e^{\lambda_{\max } t}\|\mathbf{w}(\cdot, 0)\|_{L^{2}\left(\mathbb{T}^{N}\right)},
$$

for all $t \geq 0$. 
Proof. We first consider the case for $t>1$. For any $\mathbf{q} \in \Lambda_{1}$,

$$
\left|\operatorname{det}\left[\mathbf{r}_{-}(\mathbf{q}), \mathbf{r}_{+}(\mathbf{q})\right]\right|=\frac{\lambda_{\mathbf{q}}^{+}-\lambda_{\mathbf{q}}^{-}}{\beta \tilde{u}}=\frac{\sqrt{\Delta}}{\beta \tilde{u}},
$$

where $\Delta$ is given by (14). Applying Cramer's rule to (25), we have

$$
\begin{aligned}
\left|w_{\mathbf{q}}^{ \pm}\right| & =\left|\frac{\operatorname{det}\left[\mathbf{r}_{\mp}(\mathbf{q}), \mathbf{w}_{\mathbf{q}}\right]}{\operatorname{det}\left[\mathbf{r}_{-}(\mathbf{q}), \mathbf{r}_{+}(\mathbf{q})\right]}\right| \leq \frac{\left|\mathbf{r}_{\mp}(\mathbf{q})\right| \times\left|\mathbf{w}_{\mathbf{q}}\right|}{\left|\operatorname{det}\left[\mathbf{r}_{-}(\mathbf{q}), \mathbf{r}_{+}(\mathbf{q})\right]\right|} \\
& =\frac{\beta \widetilde{u}\left|\mathbf{r}_{\mp}(\mathbf{q})\right| \times\left|\mathbf{w}_{\mathbf{q}}\right|}{\sqrt{\Delta}},
\end{aligned}
$$

where

$$
\left|\mathbf{r}_{ \pm}(\mathbf{q})\right|=\sqrt{1+\frac{\left(\lambda_{\mathbf{q}}^{ \pm}+\tilde{u}+d_{1} \mathbf{q}^{2}\right)^{2}}{(\beta \tilde{u})^{2}}} .
$$

It follows from (14) that there exist positive constants $M_{1}$ and

$$
\begin{aligned}
C_{1}= & (\widetilde{u}+\mu)\left[d_{1}+d_{2}\left(1+d_{3} \tilde{u}\right)\right] \\
& -2\left[d_{1} \mu+d_{2}\left(1+d_{3} \tilde{u}\right) \tilde{u}\right]+2 \beta d_{2} d_{3} \tilde{u} \widetilde{v}
\end{aligned}
$$

such that $\Delta>C_{1} \mathbf{q}^{2}$ for all $|\mathbf{q}|>M_{1}$. Hence, for any $|\mathbf{q}|>M_{1}$,

$$
\left|w_{\mathbf{q}}^{ \pm}\right| \leq \frac{\beta \tilde{u}\left|\mathbf{r}_{\mp}(\mathbf{q})\right| \times\left|\mathbf{w}_{\mathbf{q}}\right|}{\sqrt{C_{1}}|\mathbf{q}|}
$$

and by (12),

$$
\lim _{|\mathbf{q}| \rightarrow \infty} \frac{\lambda_{\mathbf{q}}^{ \pm}}{\mathbf{q}^{2}}=-d_{1},-d_{2}\left(1+d_{3} \tilde{u}\right)
$$

Thus,

$$
\begin{aligned}
\lim _{|\mathbf{q}| \rightarrow \infty} \frac{\left|\mathbf{r}_{ \pm}(\mathbf{q})\right|}{\mathbf{q}^{2}} & =\lim _{|\mathbf{q}| \rightarrow \infty} \frac{\left|\lambda_{\mathbf{q}}^{ \pm}+\widetilde{u}+d_{1} \mathbf{q}^{2}\right|}{\beta \tilde{u} \mathbf{q}^{2}} \\
& =\frac{1}{\beta \widetilde{u}}\left(\lim _{|\mathbf{q}| \rightarrow \infty}\left|\frac{\lambda_{\mathbf{q}}^{ \pm}}{\mathbf{q}^{2}}+d_{1}\right|\right) \\
& \leq \frac{d_{1}+d_{2}\left(1+d_{3} \tilde{u}\right)}{\beta \tilde{u}} .
\end{aligned}
$$

Consequently, there exists a positive constant $M_{2}>M_{1}$, such that

$$
\left|\mathbf{r}_{ \pm}(\mathbf{q})\right| \leq\left(\frac{d_{1}+d_{2}\left(1+d_{3} \tilde{u}\right)}{\beta \widetilde{u}}+1\right) \mathbf{q}^{2}
$$

for any $|\mathbf{q}|>M_{2}$. Substituting this into (33) yields

$$
\left|w_{\mathbf{q}}^{ \pm}\right| \leq C_{2}|\mathbf{q}|\left|\mathbf{w}_{\mathbf{q}}\right|
$$

for any $|\mathbf{q}|>M_{2}$, where $C_{2}=\left(d_{1}+d_{2}\left(1+d_{3} \tilde{u}\right)+\beta \tilde{u}\right) / \sqrt{C_{1}}$. We thus obtain

$$
\left|w_{\mathbf{q}}^{ \pm} \mathbf{r}_{ \pm}(\mathbf{q}) e^{\lambda_{\mathbf{q}}^{ \pm} t}\right| \leq C_{2}|\mathbf{q}|\left|\mathbf{w}_{\mathbf{q}}\right|\left|\mathbf{r}_{ \pm}(\mathbf{q})\right| e^{\lambda_{\mathbf{q}}^{ \pm} t} .
$$

Since

$$
\begin{aligned}
\lim _{|\mathbf{q}| \rightarrow \infty} & |\mathbf{q}|\left|\mathbf{r}_{ \pm}(\mathbf{q})\right| e^{\lambda_{\mathbf{q}}^{ \pm} t} \\
\leq & \lim _{|\mathbf{q}| \rightarrow \infty}|\mathbf{q}| \sqrt{1+\frac{\left(\lambda_{\mathbf{q}}^{ \pm}+\widetilde{u}+d_{1} \mathbf{q}^{2}\right)^{2}}{(\beta \tilde{u})^{2}}} \\
& \times e^{-\min \left\{d_{1} / 2, d_{2}\left(1+d_{3} \tilde{u}\right) / 2\right\} \mathbf{q}^{2} t} \\
= & 0,
\end{aligned}
$$

there exists a constant $M_{3}>M_{2}$, such that

$$
\left|w_{\mathbf{q}}^{ \pm} \mathbf{r}_{ \pm}(\mathbf{q}) e^{\lambda_{\mathbf{q}}^{ \pm} t}\right| \leq C_{2}\left|\mathbf{w}_{\mathbf{q}}\right|
$$

for any $|\mathbf{q}|>M_{3}$

For any $\mathbf{q} \in \Lambda_{1}$ and $|\mathbf{q}| \leq M_{3}$, as $\Delta$ is an increasing function of $|\mathbf{q}|^{2}$, we denote

$$
M_{*}=\min \{|\mathbf{q}| \Delta(\mathbf{q})>0\} .
$$

With the help of (30) and (31), we get

$$
\left|w_{\mathbf{q}}^{ \pm} \mathbf{r}_{ \pm}(\mathbf{q}) e^{\lambda_{\mathbf{q}}^{ \pm} t}\right| \leq C_{3}\left|\mathbf{w}_{\mathbf{q}}\right| e^{\lambda_{\max } t},
$$

where $C_{3}$ only depends on $\lambda, \beta, \mu, m, M_{*}$, and $M_{3}$. Hence, we conclude that, for any $\mathbf{q} \in \Lambda_{1}$, there exists a positive constant $C_{4}=\max \left\{C_{2}, C_{3}\right\}$, such that

$$
\left|w_{\mathbf{q}}^{ \pm} \mathbf{r}_{ \pm}(\mathbf{q}) e^{\lambda_{\mathbf{q}}^{ \pm} t}\right| \leq C_{4}\left|\mathbf{w}_{\mathbf{q}}\right| e^{\lambda_{\max } t}
$$

For all $\mathbf{q} \in \Lambda_{2}$ and $\mathbf{q} \in \Lambda_{3}$, by some similar arguments as above we can show that there exist positive constants $C_{5}$ and $C_{6}$, such that

$$
\begin{gathered}
\left|w_{\mathbf{q}} \mathbf{r}(\mathbf{q})\right|,\left|w_{\mathbf{q}}^{\prime} \mathbf{r}^{\prime}(\mathbf{q})\right|,\left|w_{\mathbf{q}}^{\prime} \mathbf{r}(\mathbf{q})\right| \leq C_{5}\left|\mathbf{w}_{\mathbf{q}}\right|, \quad t e^{\lambda_{\mathbf{q}} t} \leq C_{5}, \\
\left|w_{\mathbf{q}}^{\operatorname{Re}} \operatorname{Re} \mathbf{r}(\mathbf{q})\right|,\left|w_{\mathbf{q}}^{\operatorname{Re}} \operatorname{Im} \mathbf{r}(\mathbf{q})\right|,\left|w_{\mathbf{q}}^{\operatorname{Im}} \operatorname{Re} \mathbf{r}(\mathbf{q})\right|,\left|w_{\mathbf{q}}^{\operatorname{Im}} \operatorname{Im} \mathbf{r}(\mathbf{q})\right| \\
\leq C_{6}\left|\mathbf{w}_{\mathbf{q}}\right| .
\end{gathered}
$$

Next, we derive the energy estimate in $L^{2}$ for $\mathbf{w}(x, t)$. Recall that $\left\{e_{\mathbf{q}}(x)\right\}_{\mathbf{q} \in \mathbb{N}^{N}}$ is an orthonormal basis in $L^{2}\left(\mathbb{T}^{N}\right)$. Then,

$$
\|\mathbf{w}(\cdot, t)\|_{L^{2}\left(\mathbb{T}^{N}\right)}^{2}=A_{1}+A_{2}+A_{3},
$$


where

$$
\begin{aligned}
A_{1}=\sum_{\mathbf{q} \in \Lambda_{1}}\left\{w_{\mathbf{q}}^{-} \mathbf{r}_{-}(\mathbf{q}) e^{\lambda_{\mathbf{q}}^{-} t}+w_{\mathbf{q}}^{+} \mathbf{r}_{+}(\mathbf{q}) e^{\lambda_{\mathbf{q}}^{+} t}\right\}^{2} \\
A_{2}=\sum_{\mathbf{q} \in \Lambda_{2}}\left\{w_{\mathbf{q}} \mathbf{r}(\mathbf{q})+w_{\mathbf{q}}^{\prime} \mathbf{r}^{\prime}(\mathbf{q})+w_{\mathbf{q}}^{\prime} \mathbf{r}(\mathbf{q}) t\right\}^{2} e^{2 \lambda_{\mathbf{q}} t} \\
A_{3}=\sum_{\mathbf{q} \in \Lambda_{3}}\left\{w _ { \mathbf { q } } ^ { \operatorname { R e } } \left(\operatorname{Re} \mathbf{r}(\mathbf{q}) \cos \left[\left(\operatorname{Im} \lambda_{\mathbf{q}}\right) t\right]\right.\right. \\
\left.-\operatorname{Im} \mathbf{r}(\mathbf{q}) \sin \left[\left(\operatorname{Im} \lambda_{\mathbf{q}}\right) t\right]\right) \\
+w_{\mathbf{q}}^{\operatorname{Im}}\left(\operatorname{Re} \mathbf{r}(\mathbf{q}) \sin \left[\left(\operatorname{Im} \lambda_{\mathbf{q}}\right) t\right]\right. \\
\left.\left.+\operatorname{Im} \mathbf{r}(\mathbf{q}) \cos \left[\left(\operatorname{Im} \lambda_{\mathbf{q}}\right) t\right]\right)\right\}^{2} e^{2\left(\operatorname{Re} \lambda_{\mathbf{q}}\right) t}
\end{aligned}
$$

From (43) and (44), we obtain

$$
\begin{aligned}
A_{1} & \leq \sum_{\mathbf{q} \in \Lambda_{1}}\left\{\left|w_{\mathbf{q}}^{-} \mathbf{r}_{-}(\mathbf{q}) e^{\lambda_{\mathbf{q}}^{-} t}\right|+\left|w_{\mathbf{q}}^{+} \mathbf{r}_{+}(\mathbf{q}) e^{\lambda_{\mathbf{q}}^{+} t}\right|\right\}^{2} \\
& \leq 4 C_{4}^{2} \sum_{\mathbf{q} \in \Lambda_{1}}\left|\mathbf{w}_{\mathbf{q}}\right|^{2} e^{2 \lambda_{\max } t} \\
A_{2} \leq & \sum_{\mathbf{q} \in \Lambda_{2}}\left\{\left|w_{\mathbf{q}} \mathbf{r}(\mathbf{q})+w_{\mathbf{q}}^{\prime} \mathbf{r}^{\prime}(\mathbf{q})\right|+\left|w_{\mathbf{q}}^{\prime} \mathbf{r}(\mathbf{q})\right| t\right\}^{2} e^{2 \lambda_{\mathbf{q}} t} \\
\leq & 8 C_{5}^{2} \sum_{\mathbf{q} \in \Lambda_{2}}\left\{\left|\mathbf{w}_{\mathbf{q}}\right|^{2}+\left|w_{\mathbf{q}}^{\prime} \mathbf{r}(\mathbf{q})\right|^{2} t^{2}\right\} e^{2 \lambda_{\mathbf{q}} t} \\
& \leq 8 C_{5}^{2} \sum_{\mathbf{q} \in \Lambda_{2}}\left\{\left|\mathbf{w}_{\mathbf{q}}\right|^{2} e^{2 \lambda_{\mathbf{q}} t}+C_{5}^{2}\left|w_{\mathbf{q}}^{\prime} \mathbf{r}(\mathbf{q})\right|^{2}\right\} \\
\leq & 8 C_{5}^{2} \sum_{\mathbf{q} \in \Lambda_{2}}\left\{\left|\mathbf{w}_{\mathbf{q}}\right|^{2} e^{2 \lambda_{\mathbf{q}} t}+C_{5}^{4}\left|\mathbf{w}_{\mathbf{q}}\right|^{2}\right\} \\
& \leq 8 C_{5}^{2}\left(1+C_{5}^{4}\right) \sum_{\mathbf{q} \in \Lambda_{2}}\left|\mathbf{w}_{\mathbf{q}}\right|^{2} e^{2 \lambda_{\max } t}, \\
A_{3} \leq & \sum_{\mathbf{q} \in \Lambda_{3}}\left\{(|\operatorname{Re} \mathbf{r}(\mathbf{q})|+|\operatorname{Im} \mathbf{r}(\mathbf{q})|)\left(\left|w_{\mathbf{q}}^{R e}\right|+\left|w_{\mathbf{q}}^{\operatorname{Im}}\right|\right)\right\}^{2} e^{2\left(\operatorname{Re} \lambda_{\mathbf{q}}\right) t} \\
\leq & 16 C_{6}^{2} \sum_{\mathbf{q} \in \Lambda_{3}}\left|\mathbf{w}_{\mathbf{q}}\right|^{2} e^{2\left(\operatorname{Re} \lambda_{\mathbf{q}}\right) t} \\
\leq & 16 C_{6}^{2} \sum_{\mathbf{q} \in \Lambda_{3}}\left|\mathbf{w}_{\mathbf{q}}\right|^{2} e^{2 \lambda_{\max } t} .
\end{aligned}
$$

Thus,

$$
\begin{aligned}
& \|\mathbf{w}(\cdot, t)\|_{L^{2}\left(\mathbb{T}^{N}\right)}^{2} \\
& \quad \leq C_{7}^{2}\left(\sum_{\mathbf{q} \in \Lambda_{1}}\left|\mathbf{w}_{\mathbf{q}}\right|^{2}+\sum_{\mathbf{q} \in \Lambda_{2}}\left|\mathbf{w}_{\mathbf{q}}\right|^{2}+\sum_{\mathbf{q} \in \Lambda_{3}}\left|\mathbf{w}_{\mathbf{q}}\right|^{2}\right) e^{2 \lambda_{\max } t} \\
& \quad=C_{7}^{2}\|\mathbf{w}(\cdot, 0)\|_{L^{2}\left(\mathbb{T}^{N}\right)}^{2} e^{2 \lambda_{\max } t}
\end{aligned}
$$

where $C_{7}^{2}=\max \left\{4 C_{4}^{2}, 8 C_{5}^{2}\left(1+C_{5}^{4}\right), 16 C_{6}^{2}\right\}$. Finally, for any $t>$ 1 , we have

$$
\|\mathbf{w}(\cdot, t)\|_{L^{2}\left(\mathbb{T}^{N}\right)} \leq C_{7}\|\mathbf{w}(\cdot, 0)\|_{L^{2}\left(\mathbb{T}^{N}\right)} e^{\lambda_{\max } t} .
$$

For finite time $t \leq 1$, we multiply the first and second equations of (7) by $u$ and $A v$, respectively, then add them and use the integration by parts to get

$$
\begin{gathered}
\frac{1}{2} \frac{d}{d t} \int_{\mathbb{T}^{N}}\left\{|u|^{2}+A|v|^{2}\right\} d x \\
+\int_{\mathbb{T}^{N}}\left\{d_{1}|\nabla u|^{2}+A d_{2}\left(1+d_{3} \widetilde{u}\right)|\nabla v|^{2}\right. \\
\left.+A d_{2} d_{3} \widetilde{v} \nabla u \nabla v\right\} d x
\end{gathered}
$$

$$
\begin{aligned}
& +\int_{\mathbb{T}^{N}}\left\{\tilde{u} u^{2}+A \mu v^{2}\right\} d x \\
= & \int_{\mathbb{T}^{N}}\left(A \mu^{2}-\beta \tilde{u}\right) u v d x .
\end{aligned}
$$

Firstly, we claim that the integrand of the second integral in (50) satisfies

$$
\begin{gathered}
d_{1}|\nabla u|^{2}+A d_{2}\left(1+d_{3} \widetilde{u}\right)|\nabla v|^{2}+A d_{2} d_{3} \widetilde{v} \nabla u \nabla v \\
\geq \frac{1}{2}\left(d_{1}|\nabla u|^{2}+A d_{2}\left(1+d_{3} \widetilde{u}\right)|\nabla v|^{2}\right)
\end{gathered}
$$

for some positive constant $A$. Obviously, it suffices to require that

$$
\left(A d_{2} d_{3} \widetilde{v}\right)^{2}-A d_{1} d_{2}\left(1+d_{3} \widetilde{u}\right)<0 .
$$

This is equivalent to

$$
A<\frac{d_{1}\left(1+d_{3} \widetilde{u}\right)}{d_{2} d_{3}^{2} \widetilde{v}^{2}} .
$$

Denote

$$
A_{0}=\frac{d_{1}\left(1+d_{3} \widetilde{u}\right)}{2 d_{2} d_{3}^{2} \widetilde{v}^{2}} .
$$

On the other hand, the term on the right of (50) is

$$
\begin{aligned}
\int_{\mathbb{T}^{N}}\left(A \mu^{2}-\beta \tilde{u}\right) u v d x & \leq \int_{\mathbb{T}^{N}}\left(A \mu^{2}+\beta \tilde{u}\right)|u| \cdot|v| d x \\
& \leq \int_{\mathbb{T}^{N}}\left(A \mu^{2}+\beta \tilde{u}\right)\left(\frac{u^{2}+v^{2}}{2}\right) d x \\
& =\frac{A \mu^{2}+\beta \tilde{u}}{2} \int_{\mathbb{T}^{N}}\left(u^{2}+v^{2}\right) d x .
\end{aligned}
$$

Taking $A=A_{0}$, and substituting (51) and (55) into (50), we get

$$
\frac{d}{d t} \int_{\mathbb{T}^{N}}\left\{|u|^{2}+A_{0}|v|^{2}\right\} d x \leq\left(A_{0} \mu^{2}+\beta \tilde{u}\right) \int_{\mathbb{T}^{N}}\left(u^{2}+v^{2}\right) d x .
$$


Integrating (56) from 0 to $t$ leads to

$$
\begin{aligned}
\int_{\mathbb{T}^{N}} & \left\{|u(x, t)|^{2}+A_{0}|v(x, t)|^{2}\right\} d x \\
\leq & \int_{\mathbb{T}^{N}}\left\{|u(x, 0)|^{2}+A_{0}|v(x, 0)|^{2}\right\} d x \\
& +\left(A_{0} \mu^{2}+\beta \tilde{u}\right) \int_{0}^{t} \int_{\mathbb{T}^{N}}\left(u^{2}+v^{2}\right) d x d t .
\end{aligned}
$$

If $A_{0} \geq 1$, then it follows from (57) that

$$
\begin{aligned}
\int_{\mathbb{T}^{N}} & \left\{|u(x, t)|^{2}+|v(x, t)|^{2}\right\} d x \\
\leq & A_{0} \int_{\mathbb{T}^{N}}\left\{|u(x, 0)|^{2}+|v(x, 0)|^{2}\right\} d x \\
& +\left(A_{0} \mu^{2}+\beta \tilde{u}\right) \int_{0}^{t} \int_{\mathbb{T}^{N}}\left(u^{2}+v^{2}\right) d x d t
\end{aligned}
$$

thus, the Gronwall inequality implies

$$
\|\mathbf{w}(\cdot, t)\|_{L^{2}\left(\mathbb{T}^{N}\right)} \leq \sqrt{A_{0}}\|\mathbf{w}(\cdot, 0)\|_{L^{2}\left(\mathbb{T}^{N}\right)} \cdot e^{\left(\left(A_{0} \mu^{2}+\beta \widetilde{u}\right) / 2\right) t} .
$$

Consequently, there exists a positive constant $C_{8}$, such that

$$
\|\mathbf{w}(\cdot, t)\|_{L^{2}\left(\mathbb{T}^{N}\right)} \leq C_{8} \sqrt{A_{0}}\|\mathbf{w}(\cdot, 0)\|_{L^{2}\left(\mathbb{T}^{N}\right)} e^{\lambda_{\max } t}
$$

for all $t \in[0,1]$ due to the boundedness of

$$
e^{\left(\left(A_{0} \mu^{2}+\beta \tilde{u}\right) / 2-\lambda_{\max }\right) t} .
$$

If $0<A_{0}<1$, in the same way as above, there exists a positive constant $C_{9}$, such that

$$
\|\mathbf{w}(\cdot, t)\|_{L^{2}\left(\mathbb{T}^{N}\right)} \leq C_{9}\|\mathbf{w}(\cdot, 0)\|_{L^{2}\left(\mathbb{T}^{N}\right)} e^{\lambda_{\max } t} .
$$

The proof is completed by taking $\widehat{C}_{1}=\max \left\{C_{7}, C_{8} \sqrt{A_{0}}, C_{9}\right\}$.

\section{The Estimates for the Solutions of the Full System (5)}

The general theory in [21] guarantees that (5) has a unique nonnegative local solution. The results can be summarized as follows.

Lemma 2. Suppose that $\mathbf{w}(x, t)=[u, v]$ is a solution of the full system (5). For $s \geq 1(N=1)$ and $s \geq 2(N=2,3)$, there exist $a T>0$ and a constant $C$, such that

$$
\|\mathbf{w}(\cdot, t)\|_{H^{s}\left(\mathbb{T}^{N}\right)} \leq C\|\mathbf{w}(\cdot, 0)\|_{H^{s}\left(\mathbb{T}^{N}\right)}, \quad 0 \leq t<T
$$

if $u_{0}(x), v_{0}(x) \in H^{s}\left(\mathbb{T}^{N}\right)$.

Denote

$$
\partial_{i j} u:=\frac{\partial^{2} u}{\partial x_{i} \partial x_{j}}, \quad \partial_{i} u:=\frac{\partial u}{\partial x_{i}}, \quad i, j=1, \ldots, N .
$$

In order to derive the $H^{2}$ estimate for the solution of (5), we first prove the following energy estimates.
Lemma 3. Suppose that $\mathbf{w}(x, t)=[u, v]$ is a solution of the full system (5). Then,

$$
\begin{aligned}
& \frac{1}{2} \frac{d}{d t} \sum_{i, j=1}^{N} \int_{\mathbb{T}^{N}}\left\{\left|\partial_{i j} u\right|^{2}+\left|\partial_{i j} v\right|^{2}\right\} d x \\
& \quad+\sum_{i, j=1}^{N} \int_{\mathbb{T}^{N}}\left\{\frac{d_{1}}{4}\left|\nabla \partial_{i j} u\right|^{2} d x+\frac{A_{0}}{2} d_{2}\left(1+d_{3} \widetilde{u}\right)\left|\nabla \partial_{i j} v\right|^{2}\right\} d x \\
& \quad+\widetilde{u} \sum_{i, j=1}^{N} \int_{\mathbb{U}^{N}}\left|\partial_{i j} u\right|^{2} d x+\frac{A_{0} \mu}{2} \sum_{i, j=1}^{N} \int_{\mathbb{T}^{N}}\left|\partial_{i j} v\right|^{2} d x \\
& \leq \widehat{C}_{2}(1+\eta)^{2}\|\mathbf{w}(\cdot, t)\|_{H^{2}\left(\mathbb{T}^{N}\right)}\left\|\nabla^{3} \mathbf{w}(\cdot, t)\right\|_{L^{2}\left(\mathbb{T}^{N}\right)}^{2} \\
& +\widehat{C}_{2}\|u(\cdot, t)\|_{L^{2}\left(\mathbb{T}^{N}\right)}^{2}
\end{aligned}
$$

for $\|\mathbf{w}\|_{H^{2}\left(\mathbb{T}^{N}\right)} \leq \eta$.

Proof. We first notice that system (5) preserves the evenness of the solution; that is, if $\mathbf{w}\left(x_{1}, x_{2}, x_{3}, t\right)$ is a solution to (5), then $\mathbf{w}\left(-x_{1}, x_{2}, x_{3}, t\right), \mathbf{w}\left(x_{1},-x_{2}, x_{3}, t\right)$, and $\mathbf{w}\left(x_{1}, x_{2},-x_{3}, t\right)$ are also solutions of (5). We can regard system (5) as a special case with the evenness of the periodic problem by a reflective and an even extension. For this reason, we may assume periodicity at the boundary of the extended $2 \mathbb{T}^{N}=(-\pi, \pi)^{N}$. Taking the second order partial derivative of the first equation of (5), multiplying $\partial_{i j} u$, and integrating over the domain $2 \mathbb{T}^{N}$ to get

$$
\begin{aligned}
& \frac{1}{2} \frac{d}{d t} \int_{2 \mathbb{\mathbb { T }}^{N}}\left|\partial_{i j} u\right|^{2} d x \\
& \quad=\int_{2 \mathbb{T}^{N}} \partial_{i j}\left\{d_{1} \Delta u+g_{1}(u+\tilde{u}, v+\widetilde{v})\right\} \partial_{i j} u d x \\
& \quad=\int_{2 \mathbb{T}^{N}} \partial_{i j}\left\{L_{1}(u, v)+N_{1}(u, v)\right\} \partial_{i j} u d x,
\end{aligned}
$$

where

$$
\begin{gathered}
L_{1}(u, v)=d_{1} \Delta u-\tilde{u} u-\beta \tilde{u} v \\
N_{1}(u, v)=g_{1}(u+\widetilde{u}, v+\widetilde{v})+\widetilde{u} u+\beta \widetilde{u} v=-u^{2}-\beta u v
\end{gathered}
$$

are the linear and nonlinear terms, respectively, then, we have

$$
\begin{aligned}
\int_{2 \mathbb{T}^{N}} & \left(\partial_{i j} L_{1}(u, v)\right) \partial_{i j} u d x \\
= & -d_{1} \int_{2 \mathbb{T}^{N}}\left|\nabla \partial_{i j} u\right|^{2} d x-\tilde{u} \int_{2 \mathbb{T}^{N}}\left|\partial_{i j} u\right|^{2} d x \\
& -\beta \tilde{u} \int_{2 \mathbb{T}^{N}} \partial_{i j} u \partial_{i j} v d x,
\end{aligned}
$$




$$
\begin{aligned}
& \int_{2 \mathbb{J}^{N}}\left(\partial_{i j} N_{1}(u, v)\right) \partial_{i j} u d x \\
& =-\int_{2 \mathbb{T}^{N}} \partial_{i j}\left\{u^{2}+\beta u v\right\} \partial_{i j} u d x, \\
& =-\int_{2 \mathbb{J}^{N}}\left\{2 \partial_{i} u \partial_{j} u+2 u \partial_{i j} u+\beta v \partial_{i j} u+\beta \partial_{i} u \partial_{j} v\right. \\
& \left.\quad+\beta \partial_{j} u \partial_{i} v+\beta u \partial_{i j} v\right\} \partial_{i j} u d x \\
& =-\int_{2 \mathbb{T}^{N}}\left\{(2 u+\beta v) \partial_{i j} u+\beta u \partial_{i j} v\right. \\
& \left.\quad+\left(2 \partial_{i} u \partial_{j} u+\beta \partial_{i} u \partial_{j} v+\beta \partial_{j} u \partial_{i} v\right)\right\} \partial_{i j} u d x \\
& \leq \quad(2+2 \beta)\|\mathbf{w}\|_{L^{\infty}\left(2 \mathbb{T}^{N}\right)}\left\|\partial_{i j} \mathbf{w}\right\|_{L^{2}\left(2 \mathbb{T}^{N}\right)}^{2} \\
& +(2+2 \beta)\|\nabla \mathbf{w}\|_{L^{\infty}\left(2 \mathbb{T}^{N}\right)}\|\nabla \mathbf{w}\|_{L^{2}\left(2 \mathbb{T}^{N}\right)}\left\|\partial_{i j} \mathbf{w}\right\|_{L^{2}\left(2 \mathbb{T}^{N}\right)} .
\end{aligned}
$$

Similarly, taking the second order partial derivative of the second equation of (5), multiplying $\partial_{i j} v$, and integrating over the domain $2 \mathbb{T}^{N}$ to get

$$
\begin{gathered}
\frac{1}{2} \frac{d}{d t} \int_{2 \mathbb{T}^{N}}\left|\partial_{i j} v\right|^{2} d x \\
=\int_{2 \mathbb{T}^{N}} \partial_{i j}\left\{d_{2} \Delta\left[\left(1+d_{3}(u+\widetilde{u})\right)(v+\widetilde{v})\right]\right. \\
\left.\quad+g_{2}(u+\widetilde{u}, v+\widetilde{v})\right\} \partial_{i j} v d x \\
=\int_{2 \mathbb{T}^{N}} \partial_{i j}\left\{L_{2}(u, v)+N_{2}(u, v)\right\} \partial_{i j} v d x
\end{gathered}
$$

where

$$
\begin{aligned}
L_{2}(u, v)= & d_{2} d_{3} \widetilde{v} \Delta u+d_{2}\left(1+d_{3} \widetilde{u}\right) \Delta v+\mu^{2} u-\mu v, \\
N_{2}(u, v)= & d_{2} \Delta\left[\left(1+d_{3}(u+\widetilde{u})\right)(v+\widetilde{v})\right] \\
& +g_{2}(u+\widetilde{u}, v+\widetilde{v})-L_{2}(u, v) \\
= & d_{2} d_{3}(v \Delta u+2 \nabla u \nabla v+u \Delta v)-\frac{(\mu u-v)^{2}}{m+u+\widetilde{u}} \\
:= & N_{2}^{(1)}(u, v)+N_{2}^{(2)}(u, v),
\end{aligned}
$$

thus,

$$
\begin{aligned}
\int_{2 \mathbb{W}^{N}} & \left(\partial_{i j} L_{2}(u, v)\right) \partial_{i j} v d x \\
= & -d_{2} d_{3} \widetilde{v} \int_{2 \mathbb{W}^{N}} \nabla \partial_{i j} u \nabla \partial_{i j} v d x \\
& -d_{2}\left(1+d_{3} \tilde{u}\right) \int_{2 \mathbb{J}^{N}}\left|\nabla \partial_{i j} v\right|^{2} d x \\
& +\mu^{2} \int_{2 \mathbb{T}^{N}} \partial_{i j} u \partial_{i j} v d x-\mu \int_{2 \mathbb{J}^{N}}\left|\partial_{i j} v\right|^{2} d x,
\end{aligned}
$$

$$
\begin{aligned}
& \int_{2 \mathbb{T}^{N}}\left(\partial_{i j} N_{2}^{(1)}(u, v)\right) \partial_{i j} v d x \\
& =d_{2} d_{3} \int_{2 \mathbb{T}^{N}} \partial_{i j}(v \Delta u+2 \nabla u \nabla v+u \Delta v) \partial_{i j} v d x \\
& =d_{2} d_{3} \int_{2 \mathbb{T}^{N}}\left\{\left(\partial_{i j} v\right) \Delta u+v\left(\Delta \partial_{i j} u\right)+\left(\partial_{i} v\right)\left(\Delta \partial_{j} u\right)\right. \\
& \left.+\left(\partial_{j} v\right)\left(\Delta \partial_{i} u\right)\right\} \partial_{i j} v d x \\
& +2 d_{2} d_{3} \int_{2 \mathbb{T}^{N}}\left\{\left(\nabla \partial_{i j} u\right) \nabla v+\nabla u\left(\nabla \partial_{i j} v\right)\right. \\
& +\left(\nabla \partial_{i} u\right)\left(\nabla \partial_{j} v\right) \\
& \left.+\left(\nabla \partial_{j} u\right)\left(\nabla \partial_{i} v\right)\right\} \partial_{i j} v d x \\
& +d_{2} d_{3} \int_{2 \mathbb{T}^{N}}\left\{\left(\partial_{i j} u\right) \Delta v+u\left(\Delta \partial_{i j} v\right)\right. \\
& +\left(\partial_{i} u\right)\left(\Delta \partial_{j} v\right) \\
& \left.+\left(\partial_{j} u\right)\left(\Delta \partial_{i} v\right)\right\} \partial_{i j} v d x \\
& =-d_{2} d_{3} \int_{2 \mathbb{T}^{N}}\left(\partial_{i j} v\right)\left(\nabla \partial_{i j} v\right) \nabla u d x \\
& -d_{2} d_{3} \int_{2 \mathbb{\mathbb { T }}^{N}} \nabla v\left(\partial_{i j} u\right)\left(\nabla \partial_{i j} v\right) d x \\
& -d_{2} d_{3} \int_{2 \pi^{N}} v\left(\nabla \partial_{i j} v\right)\left(\nabla \partial_{i j} u\right) d x+d_{2} d_{3} \\
& \times \int_{2 \mathbb{\pi}^{N}}\left\{\left(\partial_{i} v\right)\left(\Delta \partial_{j} u\right)+\left(\partial_{j} v\right)\left(\Delta \partial_{i} u\right)\right\} \partial_{i j} v d x \\
& -2 d_{2} d_{3} \int_{2 \mathbb{\pi}^{N}}\left\{\left(\Delta \partial_{i} u\right) \partial_{i j} v \partial_{j} v\right. \\
& +\left(\nabla \partial_{i} u\right)\left(\nabla \partial_{i j} v\right) \partial_{j} v \\
& +\left(\Delta \partial_{j} u\right) \partial_{i j} v \partial_{i} v \\
& \left.+\left(\nabla \partial_{j} u\right)\left(\nabla \partial_{i j} v\right) \partial_{i} v\right\} d x \\
& -d_{2} d_{3} \int_{2 \mathbb{\boxplus}^{N}} u\left|\nabla \partial_{i j} v\right|^{2} d x+d_{2} d_{3} \\
& \times \int_{2 \mathbb{T}^{N}}\left\{\left(\partial_{i} u\right)\left(\Delta \partial_{j} v\right)+\left(\partial_{j} u\right)\left(\Delta \partial_{i} v\right)\right\} \partial_{i j} v d x \\
& \leq 14 d_{2} d_{3}\|\nabla \mathbf{w}\|_{L^{\infty}\left(2 \mathbb{T}^{N}\right)}\left\|\partial_{i j} \mathbf{w}\right\|_{L^{2}\left(2 \mathbb{T}^{N}\right)}\left\|\nabla^{3} \mathbf{w}\right\|_{L^{2}\left(2 \mathbb{T}^{N}\right)} \\
& +2 d_{2} d_{3}\|\mathbf{w}\|_{L^{\infty}\left(2 \mathbb{T}^{N}\right)}\left\|\nabla^{3} \mathbf{w}\right\|_{L^{2}\left(2 \mathbb{T}^{N}\right)}^{2}, \\
& \int_{2 \mathbb{\Psi}^{N}}\left(\partial_{i j} N_{2}^{(2)}(u, v)\right) \partial_{i j} v d x \\
& =-\int_{2 \mathbb{T}^{N}} \partial_{i j}\left(\frac{(\mu u-v)^{2}}{m+u+\widetilde{u}}\right) \partial_{i j} v d x
\end{aligned}
$$




$$
\begin{aligned}
& =\int_{2 \mathbb{\mathbb { T }}^{N}}\left\{-\left(2(\mu u-v)\left(\mu \partial_{i j} u-\partial_{i j} v\right)\right.\right. \\
& \left.+2\left(\mu \partial_{i} u-\partial_{i} v\right)\left(\mu \partial_{j} u-\partial_{j} v\right)\right) \times(m+u+\widetilde{u})^{-1} \\
& -(\mu u-v)^{2}\left[\frac{2 \partial_{i} u \partial_{j} u}{(m+u+\widetilde{u})^{3}}-\frac{\partial_{i j} u}{(m+u+\widetilde{u})^{2}}\right] \\
& +\left(2 ( \mu u - v ) \left[\left(\mu \partial_{i} u-\partial_{i} v\right) \partial_{j} u\right.\right. \\
& \left.\left.+\left(\mu \partial_{j} u-\partial_{j} v\right) \partial_{i} u\right]\right) \\
& \left.\times(m+u+\widetilde{u})^{-2}\right\} \partial_{i j} v d x \\
& \leq \frac{2(\mu+1)^{2}}{m}\|\mathbf{w}\|_{L^{\infty}\left(2 \mathbb{T}^{N}\right)}\left\|\partial_{i j} \mathbf{w}\right\|_{L^{2}\left(2 \mathbb{T}^{N}\right)}^{2} \\
& +\frac{2(\mu+1)^{2}}{m}\|\nabla \mathbf{w}\|_{L^{\infty}\left(2 \mathbb{J}^{N}\right)}\|\nabla \mathbf{w}\|_{L^{2}\left(2 \mathbb{T}^{N}\right)}\left\|\partial_{i j} \mathbf{w}\right\|_{L^{2}\left(2 \mathbb{T}^{N}\right)} \\
& +\frac{2(\mu+1)^{2}}{m^{3}}\|\mathbf{w}\|_{L^{\infty}\left(2 \mathbb{T}^{N}\right)}^{2}\|\nabla \mathbf{w}\|_{L^{\infty}\left(2 \mathbb{T}^{N}\right)} \\
& \times\|\nabla \mathbf{w}\|_{L^{2}\left(2 \mathbb{T}^{N}\right)}\left\|\partial_{i j} \mathbf{w}\right\|_{L^{2}\left(2 \mathbb{T}^{N}\right)} \\
& +\frac{(\mu+1)^{2}}{m^{2}}\|\mathbf{w}\|_{L^{\infty}\left(2 \mathbb{J}^{N}\right)}^{2}\left\|\partial_{i j} \mathbf{w}\right\|_{L^{2}\left(2 \mathbb{J}^{N}\right)}^{2} \\
& +\frac{4(\mu+1)^{2}}{m^{2}}\|\mathbf{w}\|_{L^{\infty}\left(2 \mathbb{J}^{N}\right)}\|\nabla \mathbf{w}\|_{L^{\infty}\left(2 \mathbb{J}^{N}\right)} \\
& \times\|\nabla \mathbf{w}\|_{L^{2}\left(2 \mathbb{T}^{N}\right)}\left\|\partial_{i j} \mathbf{w}\right\|_{L^{2}\left(2 \mathbb{T}^{N}\right)} \\
& \leq \frac{(\mu+1)^{2}}{m}\left(2+\frac{\|\mathbf{w}\|_{L^{\infty}\left(2 \mathbb{J}^{N}\right)}}{m}\right)\|\mathbf{w}\|_{L^{\infty}\left(2 \mathbb{J}^{N}\right)}\left\|\partial_{i j} \mathbf{w}\right\|_{L^{2}\left(2 \mathbb{T}^{N}\right)}^{2} \\
& +\frac{2(\mu+1)^{2}}{m}\left(1+\frac{\|\mathbf{w}\|_{L^{\infty}\left(2 \mathbb{J}^{N}\right)}}{m}\right)^{2}\|\nabla \mathbf{w}\|_{L^{\infty}\left(2 \mathbb{J}^{N}\right)} \\
& \times\|\nabla \mathbf{w}\|_{L^{2}\left(2 \mathbb{J}^{N}\right)}\left\|\partial_{i j} \mathbf{w}\right\|_{L^{2}\left(2 \mathbb{T}^{N}\right)} .
\end{aligned}
$$

Substituting (70) $\times A_{0}+(66)$ into (69) and (73) to get

$$
\begin{aligned}
& \frac{1}{2} \frac{d}{d t} \int_{2 \mathbb{T}^{N}}\left\{\left|\partial_{i j} u\right|^{2}+A_{0}\left|\partial_{i j} v\right|^{2}\right\} d x \\
& +\int_{2 \mathbb{T}^{N}}\left\{d_{1}\left|\nabla \partial_{i j} u\right|^{2}+A_{0} d_{2} d_{3} \widetilde{v} \nabla \partial_{i j} u \nabla \partial_{i j} v\right. \\
& \left.\quad+A_{0} d_{2}\left(1+d_{3} \widetilde{u}\right)\left|\nabla \partial_{i j} v\right|^{2}\right\} d x \\
& +\widetilde{u} \int_{2 \mathbb{T}^{N}}\left|\partial_{i j} u\right|^{2} d x+A_{0} \mu \int_{2 \mathbb{T}^{N}}\left|\partial_{i j} v\right|^{2} d x \\
& \leq I_{1}+I_{2},
\end{aligned}
$$

where

$$
\begin{aligned}
I_{1}= & C_{10}(1+\eta)\|\mathbf{w}\|_{L^{\infty}\left(2 \mathbb{T}^{N}\right)}\left\|\partial_{i j} \mathbf{w}\right\|_{L^{2}\left(2 \mathbb{T}^{N}\right)}^{2} \\
& +C_{11}(1+\eta)^{2}\|\nabla \mathbf{w}\|_{L^{\infty}\left(2 \mathbb{T}^{N}\right)}\|\nabla \mathbf{w}\|_{L^{2}\left(2 \mathbb{T}^{N}\right)}\left\|\partial_{i j} \mathbf{w}\right\|_{L^{2}\left(2 \mathbb{T}^{N}\right)} \\
& +C_{12}\|\nabla \mathbf{w}\|_{L^{\infty}\left(2 \mathbb{T}^{N}\right)}\left\|\partial_{i j} \mathbf{w}\right\|_{L^{2}\left(2 \mathbb{T}^{N}\right)}\left\|\nabla^{3} \mathbf{w}\right\|_{L^{2}\left(2 \mathbb{J}^{N}\right)} \\
& +C_{13}\|\mathbf{w}\|_{L^{\infty}\left(2 \mathbb{T}^{N}\right)}\left\|\nabla^{3} \mathbf{w}\right\|_{L^{2}\left(2 \mathbb{T}^{N}\right)}^{2}, \\
I_{2}= & \left(A_{0} \mu^{2}-\beta \widetilde{u}\right) \int_{2 \mathbb{T}^{N}} \partial_{i j} u \partial_{i j} v d x,
\end{aligned}
$$

we apply the Sobolev imbedding to control the $L^{\infty}$ norm by

$$
\|g\|_{L^{\infty}\left(2 \mathbb{T}^{N}\right)} \leq C_{0}\|g\|_{H^{2}\left(2 \mathbb{J}^{N}\right)}
$$

for $N \leq 3$. From the Hölder inequality, the Poincare inequality, and the Sobolev imbedding theorem, it follows that

$$
\|g\|_{L^{2}\left(2 \mathbb{T}^{N}\right)} \leq(2 \pi)^{N / 4}\|g\|_{L^{4}\left(2 \mathbb{T}^{N}\right)} \leq C_{0}\|\nabla g\|_{L^{2}\left(2 \mathbb{T}^{N}\right)}
$$

for $\int_{2 \pi^{N}} g d x=0$. Recall the even extension of (5), and the solution $[u, v]$ satisfies

$$
\begin{aligned}
& \int_{2 \mathbb{\pi}^{N}} \nabla u d x=\int_{2 \mathbb{\pi}^{N}} \nabla v d x=0, \\
& \int_{2 \mathbb{\pi}^{N}} \partial_{i j} u d x=\int_{2 \mathbb{\pi}^{N}} \partial_{i j} v d x=0, \quad i, j=1, \ldots, N .
\end{aligned}
$$

By (76) and (77), we find that

$$
\|\nabla \mathbf{w}\|_{L^{\infty}\left(2 \mathbb{T}^{N}\right)} \leq C_{0}\|\nabla \mathbf{w}\|_{H^{2}\left(2 \mathbb{J}^{N}\right)} \leq C_{0}\left\|\nabla^{3} \mathbf{w}\right\|_{L^{2}\left(2 \mathbb{T}^{N}\right)},
$$

where $C_{0}$ is a universal constant. Therefore, when $N \leq 3$, it follows from (76) and (79) that

$$
I_{1} \leq C_{14}(1+\eta)^{2}\|\mathbf{w}\|_{H^{2}\left(2 \mathbb{T}^{N}\right)}\left\|\nabla^{3} \mathbf{w}\right\|_{L^{2}\left(2 \mathbb{T}^{N}\right)}^{2} .
$$

Applying the Young inequality to get

$$
I_{2} \leq \frac{\left(A_{0} \mu^{2}+\beta \tilde{u}\right)^{2}}{2 A_{0} \mu} \int_{2 \mathbb{T}^{N}}\left|\partial_{i j} u\right|^{2} d x+\frac{A_{0} \mu}{2} \int_{2 \mathbb{T}^{N}}\left|\partial_{i j} v\right|^{2} d x,
$$

which is combined with the interpolation inequality and the $\varepsilon$-Young inequality to imply

$$
\int_{2 \mathbb{\Psi}^{N}}\left|\partial_{i j} u\right|^{2} d x \leq C_{0}\left(a\left\|\nabla \partial_{i j} u\right\|_{L^{2}\left(2 \mathbb{J}^{N}\right)}^{2}\right)+\frac{1}{4 a^{2}}\|u\|_{L^{2}\left(2 \mathbb{J}^{N}\right)}^{2},
$$

where $a$ is a positive constant, in the same way as above, we obtain that the second integral satisfies

$$
\begin{aligned}
\int_{2 \mathbb{T}^{N}} & \left\{d_{1}\left|\nabla \partial_{i j} u\right|^{2}+A_{0} d_{2} d_{3} \widetilde{v} \nabla \partial_{i j} u \nabla \partial_{i j} v\right. \\
& \left.+A_{0} d_{2}\left(1+d_{3} \widetilde{u}\right)\left|\nabla \partial_{i j} v\right|^{2}\right\} d x \\
\geq & \left\{\frac{d_{1}}{2}\left|\nabla \partial_{i j} u\right|^{2}+\frac{A_{0} d_{2}\left(1+d_{3} \widetilde{u}\right)}{2}\left|\nabla \partial_{i j} v\right|^{2}\right\} d x .
\end{aligned}
$$


Substituting (80)-(83) into (74), take $\left(\left(A_{0} \mu^{2}+\beta \widetilde{u}\right)^{2} / 2 A_{0} \mu\right)$ $C_{0} a=d_{1} / 4$ to get

$$
\begin{aligned}
\frac{1}{2} \frac{d}{d t} \int_{2 \mathbb{T}^{N}} & \left\{\left|\partial_{i j} u\right|^{2}+A_{0}\left|\partial_{i j} v\right|^{2}\right\} d x \\
& +\int_{2 \mathbb{J}^{N}}\left\{\frac{d_{1}}{4}\left|\nabla \partial_{i j} u\right|^{2}+\frac{A_{0}}{2} d_{2}\left(1+d_{3} \tilde{u}\right)\left|\nabla \partial_{i j} v\right|^{2}\right\} d x \\
& +\widetilde{u} \int_{2 \mathbb{T}^{N}}\left|\partial_{i j} u\right|^{2} d x+\frac{A_{0} \mu}{2} \int_{2 \mathbb{T}^{N}}\left|\partial_{i j} v\right|^{2} d x \\
\leq & C_{14}(1+\eta)^{2}\|\mathbf{w}\|_{H^{2}\left(2 \mathbb{T}^{N}\right)}\left\|\nabla^{3} \mathbf{w}\right\|_{L^{2}\left(2 \mathbb{T}^{N}\right)}^{2} \\
& +C_{15}\|u\|_{L^{2}\left(2 \mathbb{J}^{N}\right)}^{2} .
\end{aligned}
$$

Similar as the proof of Lemma 1, we proceed in the two cases: $A_{0} \geq 1$ and $0<A_{0}<1$. Then, we conclude

$$
\begin{aligned}
\frac{1}{2} \frac{d}{d t} \int_{2 \mathbb{T}^{N}} & \left\{\left|\partial_{i j} u\right|^{2}+\left|\partial_{i j} v\right|^{2}\right\} d x \\
& +\int_{2 \mathbb{J}^{N}}\left\{\frac{d_{1}}{4}\left|\nabla \partial_{i j} u\right|^{2}+\frac{A_{0}}{2} d_{2}\left(1+d_{3} \tilde{u}\right)\left|\nabla \partial_{i j} v\right|^{2}\right\} d x \\
& +\tilde{u} \int_{2 \mathbb{T}^{N}}\left|\partial_{i j} u\right|^{2} d x+\frac{A_{0} \mu}{2} \int_{2 \mathbb{T}^{N}}\left|\partial_{i j} v\right|^{2} d x \\
\leq & C_{16}(1+\eta)^{2}\|\mathbf{w}\|_{H^{2}\left(2 \mathbb{T}^{N}\right)}\left\|\nabla^{3} \mathbf{w}\right\|_{L^{2}\left(2 \mathbb{T}^{N}\right)}^{2} \\
& +C_{17}\|u\|_{L^{2}\left(2 \mathbb{T}^{N}\right)}^{2} .
\end{aligned}
$$

So, the even extension implies

$$
\begin{aligned}
& \frac{1}{2} \frac{d}{d t} \sum_{i, j=1}^{N} \int_{\mathbb{T}^{N}}\left\{\left|\partial_{i j} u\right|^{2}+\left|\partial_{i j} v\right|^{2}\right\} d x \\
& \quad+\sum_{i, j=1}^{N} \int_{\mathbb{T}^{N}}\left\{\frac{d_{1}}{4}\left|\nabla \partial_{i j} u\right|^{2}+\frac{A_{0}}{2} d_{2}\left(1+d_{3} \widetilde{u}\right)\left|\nabla \partial_{i j} v\right|^{2}\right\} d x \\
& \quad+\widetilde{u} \sum_{i, j=1}^{N} \int_{\mathbb{T}^{N}}\left|\partial_{i j} u\right|^{2} d x+\frac{A_{0} \mu}{2} \sum_{i, j=1}^{N} \int_{\mathbb{T}^{N}}\left|\partial_{i j} v\right|^{2} d x \\
& \leq \widehat{C}_{2}(1+\eta)^{2}\|\mathbf{w}\|_{H^{2}\left(\mathbb{T}^{N}\right)}\left\|\nabla^{3} \mathbf{w}\right\|_{L^{2}\left(\mathbb{T}^{N}\right)}^{2}+\widehat{C}_{2}\|u\|_{L^{2}\left(\mathbb{T}^{N}\right)}^{2} .
\end{aligned}
$$

Next, we control the $H^{2}$ growth of $\mathbf{w}(x, t)$ in terms of its $L^{2}$ growth.

Lemma 4. Suppose that $\mathbf{w}(x, t)$ is a solution of the full system (5), such that

$$
\|\mathbf{w}(\cdot, t)\|_{H^{2}\left(\mathbb{T}^{N}\right)} \leq \min \left\{\eta, \frac{d_{1}}{4 \widehat{C}_{2}(1+\eta)^{2}}, \frac{A_{0} d_{2}\left(1+d_{3} \tilde{u}\right)}{2 \widehat{C}_{2}(1+\eta)^{2}}\right\},
$$

$$
\|\mathbf{w}(\cdot, t)\|_{L^{2}\left(\mathbb{T}^{N}\right)} \leq 2 \widehat{C}_{1} e^{\lambda_{\max } t}\|\mathbf{w}(\cdot, 0)\|_{L^{2}\left(\mathbb{T}^{N}\right)} .
$$

Then,

$$
\begin{aligned}
\|\mathbf{w}(\cdot, t)\|_{H^{2}\left(\mathbb{T}^{N}\right)}^{2} \leq \widehat{C}_{3}\{ & \|\mathbf{w}(\cdot, 0)\|_{H^{2}\left(\mathbb{T}^{N}\right)}^{2} \\
& \left.+e^{2 \lambda_{\max } t}\|\mathbf{w}(\cdot, 0)\|_{L^{2}\left(\mathbb{T}^{N}\right)}^{2}\right\},
\end{aligned}
$$

where $\widehat{C}_{3}=2 \max \left\{4 \widehat{C}_{1}^{2}, 4\left(C_{0}+1\right) \widehat{C}_{1}^{2} \widehat{C}_{2} / \lambda_{\max },\left(C_{0}+1\right) / 2\right\}$.

Proof. We first consider the second-order derivatives of $\mathbf{w}(x, t)$. From Lemmas 3 and 4 and our assumption for $\mathbf{w}(x, t)$, we have

$$
\begin{gathered}
\frac{1}{2} \frac{d}{d t} \sum_{i, j=1}^{N} \int_{\mathbb{T}^{N}}\left\{\left|\partial_{i j} u\right|^{2}+\left|\partial_{i j} v\right|^{2}\right\} d x \leq \widehat{C}_{2}\|u\|_{L^{2}\left(\mathbb{T}^{N}\right)}^{2} \\
\leq 4 \widehat{C}_{1}^{2} \widehat{C}_{2} e^{2 \lambda_{\max } t}\|\mathbf{w}(\cdot, 0)\|_{L^{2}\left(\mathbb{T}^{N}\right)}^{2} .
\end{gathered}
$$

By an integration from 0 to $t$, we deduce that

$$
\begin{aligned}
& \sum_{i, j=1}^{N} \int_{\mathbb{T}^{N}}\left\{\left|\partial_{i j} u(x, t)\right|^{2}+\left|\partial_{i j} v(x, t)\right|^{2}\right\} d x \\
& \leq \sum_{i, j=1}^{N} \int_{\mathbb{T}^{N}}\left\{\left|\partial_{i j} u(x, 0)\right|^{2}+\left|\partial_{i j} v(x, 0)\right|^{2}\right\} d x \\
& +\frac{4 \widehat{C}_{1}^{2} \widehat{C}_{2}}{\lambda_{\max }} e^{2 \lambda_{\max } t}\|\mathbf{w}(\cdot, 0)\|_{L^{2}\left(\mathbb{T}^{N}\right)}^{2}
\end{aligned}
$$

For the first derivations of $\mathbf{w}(x, t)$, we apply the Poincare inequality to get

$$
\|\nabla \mathbf{w}\|_{L^{2}\left(2 \mathbb{T}^{N}\right)} \leq C_{0}\left\|\nabla^{2} \mathbf{w}\right\|_{L^{2}\left(2 \mathbb{J}^{N}\right)} .
$$

Applying the even extension, we have

$$
\|\nabla \mathbf{w}\|_{L^{2}\left(\mathbb{T}^{N}\right)} \leq C_{0}\left\|\nabla^{2} \mathbf{w}\right\|_{L^{2}\left(\mathbb{T}^{N}\right)} .
$$

It follows from (88)-(93) that

$$
\begin{aligned}
&\|\mathbf{w}(\cdot, \mathbf{t})\|_{H^{2}\left(\mathbb{T}^{N}\right)}^{2}\|\mathbf{w}(\cdot, \mathbf{t})\|_{L^{2}\left(\mathbb{T}^{N}\right)}^{2}+\|\nabla \mathbf{w}\|_{L^{2}\left(\mathbb{T}^{N}\right)}^{2}+\left\|\nabla^{2} \mathbf{w}\right\|_{L^{2}\left(\mathbb{T}^{N}\right)}^{2} \\
& \leq\|\mathbf{w}(\cdot, \mathbf{t})\|_{L^{2}\left(\mathbb{T}^{N}\right)}^{2}+\left(C_{0}+1\right)\left\|\nabla^{2} \mathbf{w}\right\|_{L^{2}\left(\mathbb{T}^{N}\right)}^{2} \\
& \leq 4 \widehat{C}_{1}^{2} e^{2 \lambda_{\max } t}\|\mathbf{w}(\cdot, \mathbf{0})\|_{L^{2}\left(\mathbb{T}^{N}\right)}^{2} \\
&+\left(C_{0}+1\right)\|\mathbf{w}(\cdot, \mathbf{0})\|_{H^{2}\left(\mathbb{T}^{N}\right)}^{2} \\
&+\frac{4\left(C_{0}+1\right) \widehat{C}_{1}^{2} \widehat{C}_{2}}{\lambda_{\max }} e^{2 \lambda_{\max } t}\|\mathbf{w}(\cdot, 0)\|_{L^{2}\left(\mathbb{T}^{N}\right)}^{2} \\
& \leq \widehat{C}_{3}\left\{\|\mathbf{w}(\cdot, \mathbf{0})\|_{H^{2}\left(\mathbb{T}^{N}\right)}^{2}+e^{2 \lambda_{\max } t}\|\mathbf{w}(\cdot, \mathbf{0})\|_{L^{2}\left(\mathbb{T}^{N}\right)}^{2}\right\}, \\
& \text { where } \widehat{C}_{3}=2 \max \left\{4 \widehat{C}_{1}^{2}, 4\left(C_{0}+1\right) \widehat{C}_{1}^{2} \widehat{C}_{2} / \lambda_{\max },\left(C_{0}+1\right) / 2\right\} .
\end{aligned}
$$




\section{Nonlinear Instability and Pattern Formation}

Let $\theta$ be a fixed constant. For an arbitrary positive number $\delta$, we define the escape time $T^{\delta}$ by

$$
\theta=\delta e^{\lambda_{\max } T^{\delta}}
$$

or equivalently

$$
T^{\delta}=\frac{1}{\lambda_{\max }} \ln \frac{\theta}{\delta} .
$$

Theorem 5. Assume that there exists a q, such that the instability criterion (13) holds. Let

$$
\begin{aligned}
\mathbf{w}_{0}(x)= & \sum_{\mathbf{q} \in \Lambda_{1}}\left\{w_{\mathbf{q}}^{-} \mathbf{r}_{-}(\mathbf{q})+w_{\mathbf{q}}^{+} \mathbf{r}_{+}(\mathbf{q})\right\} e_{\mathbf{q}}(x) \\
& +\sum_{\mathbf{q} \in \Lambda_{2}}\left\{w_{\mathbf{q}} \mathbf{r}(\mathbf{q})+w_{\mathbf{q}}^{\prime} \mathbf{r}^{\prime}(\mathbf{q})\right\} e_{\mathbf{q}}(x) \\
& +\sum_{\mathbf{q} \in \Lambda_{3}}\left\{w_{\mathbf{q}}^{\operatorname{Re}} \operatorname{Re} \mathbf{r}(\mathbf{q})+w_{\mathbf{q}}^{\operatorname{Im}} \operatorname{Im} \mathbf{r}(\mathbf{q})\right\} e_{\mathbf{q}}(x),
\end{aligned}
$$

such that $\mathbf{w}_{0}(x) \in H^{2}\left(\mathbb{T}^{N}\right),\left\|\mathbf{w}_{0}\right\|_{L^{2}\left(\mathbb{T}^{N}\right)}=1$. Then, there exist $\theta_{0}>0, \delta_{0}>0$, and $C>0$, such that for all $\theta \in\left(0, \theta_{0}\right)$ and $\delta \epsilon$ $\left(0, \delta_{0}\right]$, the nonlinear evolution $\mathbf{w}^{\delta}(x, t)$ of $(5)$ with the initial perturbation $\mathbf{w}^{\delta}(x, 0)=\delta \mathbf{w}_{0}(x)$ satisfies

$$
\begin{array}{r}
\left\|\mathbf{w}^{\delta}(\cdot, t)-\delta e^{\lambda_{\max } t} \sum_{\mathbf{q} \in \Lambda_{\max }} w_{\mathbf{q}}^{+} \mathbf{r}_{+}(\mathbf{q}) e_{\mathbf{q}}(x)\right\|_{L^{2}\left(\mathbb{T}^{N}\right)} \\
\leq C\left\{e^{-\sigma t}+\delta\left\|\mathbf{w}_{\mathbf{0}}\right\|_{H^{2}\left(\mathbb{T}^{N}\right)}^{2}+\delta e^{\lambda_{\max } t}\right\} \delta e^{\lambda_{\max } t}, \\
\forall t \in\left[0, T^{\delta}\right],
\end{array}
$$

where the constant $\sigma$, called the gap between $\lambda_{\max }$ and the rest of eigenvalues, is positive, and

$$
\begin{aligned}
& \delta_{0}=\min \left\{\frac{1}{2 \sqrt{\widehat{C}_{3}}\left\|\mathbf{w}_{0}\right\|_{H^{2}\left(T^{N}\right)}},\right. \\
& \min \left\{\eta, \frac{d_{1}}{4 \widehat{C}^{2}(1+\eta)^{2}}, \frac{A_{0} d_{2}\left(1+d_{3} \widetilde{u}\right)}{2 \widehat{C}_{1}(1+\eta)^{2}}\right\}, \\
&\left.\frac{\lambda_{\max }}{4 \widehat{C}_{3} \widehat{C}_{4}\left\|\mathbf{w}_{0}\right\|_{H^{2}\left(T^{N}\right)}^{2}}\right\}, \\
& \theta_{0}=\min \left\{\frac{\lambda_{\max }}{4 \widehat{C}_{3} \widehat{C}_{4}}, \frac{\eta}{2 \sqrt{\widehat{C}_{3}}},\right. \\
&\left.\frac{d_{1}}{8 \widehat{C}_{2} \sqrt{\widehat{C}_{3}}(1+\eta)^{2}}, \frac{A_{0} d_{2}\left(1+d_{3} \widetilde{u}\right)}{4 \widehat{C}_{2} \sqrt{\widehat{C}_{3}}(1+\eta)^{2}}\right\} .
\end{aligned}
$$

Remark 6. First, we notice that the part of the fastest growing modes

$$
\delta e^{\lambda_{\max } t} \sum_{\mathbf{q} \in \Lambda_{\max }} w_{\mathbf{q}}^{+} \mathbf{r}_{+}(\mathbf{q}) e_{\mathbf{q}}(x)
$$

in (98) is nontrivial. Recall that the initial profile $\mathbf{w}_{0}(x)$ is any $H^{2}$ function satisfying $\left\|\mathbf{w}_{\mathbf{0}}\right\|_{L^{2}\left(\mathbb{T}^{N}\right)}=1$. Thus, we can choose $\mathbf{w}_{0}(x)$, such that there is at least one $\mathbf{q}_{\mathbf{0}} \in \Lambda_{\max }$ with $w_{\mathbf{q}_{0}}^{+} \neq 0$. Consequently,

$$
\begin{array}{r}
\left\|\delta e^{\lambda_{\max } t} \sum_{\mathbf{q} \in \Lambda_{\text {max }}} w_{\mathbf{q}}^{+} \mathbf{r}_{+}(\mathbf{q}) e_{\mathbf{q}}(x)\right\|_{L^{2}\left(\mathbb{T}^{N}\right)} \\
\geq \delta e^{\lambda_{\text {max }} t}\left|w_{\mathbf{q}_{0}}^{+}\right|\left|\mathbf{r}_{+}\left(\mathbf{q}_{\mathbf{0}}\right)\right|>0 .
\end{array}
$$

Remark 7. Fix $\theta$ to be a positive small number. If $\delta$ is small sufficiently, then $T^{\delta}$ is large, and for $0 \leq t \leq T^{\delta}, \delta e^{\lambda_{\max }{ }^{t}} \leq$ $\theta$. Our estimate (98) implies that the dynamics of a general perturbation can be characterized by such linear dynamics over a long time period $\left(0, T^{\delta}\right]$, when the initial perturbation is small.

Remark 8. In particular, if we take

$$
\mathbf{w}_{0}(x)=\frac{\mathbf{r}_{+}\left(\mathbf{q}_{\mathbf{0}}\right)}{\left|\mathbf{r}_{+}\left(\mathbf{q}_{\mathbf{0}}\right)\right|} e_{\mathbf{q}_{\mathbf{0}}}(x)
$$

in Remark 6, then, at the time $t=T^{\delta}$, the estimate (98) gives

$$
\begin{aligned}
\left\|\mathbf{w}^{\delta}\left(\cdot, T^{\delta}\right)-\delta e^{\lambda_{\max } T^{\delta}} \frac{\mathbf{r}_{+}\left(\mathbf{q}_{\mathbf{0}}\right)}{\left|\mathbf{r}_{+}\left(\mathbf{q}_{\mathbf{0}}\right)\right|} e_{\mathbf{q}_{\mathbf{0}}}(\cdot)\right\|_{L^{2}\left(\mathbb{T}^{N}\right)} \\
\quad \leq C\left\{e^{-\sigma T^{\delta}}+\delta\left\|\mathbf{w}_{\mathbf{0}}\right\|_{H^{2}\left(\mathbb{T}^{N}\right)}^{2}+\theta\right\} \theta, \\
\quad=C\left\{\theta^{1-\sigma / \lambda_{\max }} \delta^{\sigma / \lambda_{\max }}+\delta \theta\left\|\mathbf{w}_{\mathbf{0}}\right\|_{H^{2}\left(\mathbb{T}^{N}\right)}^{2}+\theta^{2}\right\} .
\end{aligned}
$$

Therefore,

$$
\begin{aligned}
&\left\|\mathbf{w}^{\delta}\left(\cdot, T^{\delta}\right)\right\| \geq \theta-C\left\{\theta^{1-\sigma / \lambda_{\max }} \delta^{\sigma / \lambda_{\max }}\right. \\
&\left.+\delta \theta\left\|\mathbf{w}_{\mathbf{0}}\right\|_{H^{2}\left(\mathbb{T}^{N}\right)}^{2}+\theta^{2}\right\} .
\end{aligned}
$$

For $\theta$ sufficiently small, $\left\|\mathbf{w}^{\delta}\left(., T^{\delta}\right)\right\| \geq \theta / 2$ as $\delta \rightarrow 0$, which implies that nonlinear instability occurs.

Remark 9. From a view of pattern formation, Theorem 5 shows that if the unique positive equilibrium $(\widetilde{u}, \widetilde{v})$ of $(2)$ is linear unstable, then a general small perturbation near $(\widetilde{u}, \widetilde{v})$ can induce pattern formation. Furthermore, the patterns can be characterized by the fastest growing modes in the corresponding linear dynamics over a long time period $\left(0, T^{\delta}\right]$. 
Proof of Theorem 5. Define

$$
\begin{gathered}
T^{*}=\sup \left\{t \mid\left\|\mathbf{w}^{\delta}(\cdot, t)-\delta e^{L t} \mathbf{w}_{0}\right\|_{L^{2}\left(\mathbb{T}^{N}\right)} \leq \frac{\widehat{C}_{1}}{2} \delta e^{\lambda_{\max } t}\right\}, \\
T^{* *}=\sup \left\{t \mid\left\|\mathbf{w}^{\delta}(\cdot, t)\right\|_{H^{2}\left(\mathbb{T}^{N}\right)}\right. \\
\left.\leq \min \left\{\eta, \frac{d_{1}}{4 \widehat{C}_{2}(1+\eta)^{2}}, \frac{A_{0} d_{2}\left(1+d_{3} \widetilde{u}\right)}{2 \widehat{C}_{2}(1+\eta)^{2}}\right\}\right\} .
\end{gathered}
$$

Now, we proceed in the following four steps.

Step 1. We establish $H^{2}$ estimate for the solution $\mathbf{w}^{\delta}(x, t)$ of $0 \leq t \leq \min \left\{T^{\delta}, T^{*}, T^{* *}\right\}$.

From Lemma 1 , for any $t \geq 0$, we have

$$
\left\|\delta e^{L t} \mathbf{w}_{0}\right\|_{L^{2}\left(\mathbb{T}^{N}\right)} \leq \delta \widehat{C}_{1} e^{\lambda_{\max } t} .
$$

By the definition of $T^{*}$, for any $0 \leq t \leq T^{*}$, it follows that

$$
\left\|\mathbf{w}^{\delta}(\cdot, t)\right\|_{L^{2}\left(\mathbb{T}^{N}\right)}-\left\|\delta e^{L t} \mathbf{w}_{0}\right\|_{L^{2}\left(\mathbb{T}^{N}\right)} \leq \frac{\widehat{C}_{1}}{2} \delta e^{\lambda_{\max } t}
$$

Substituting (107) into (108), we obtain

$$
\left\|\mathbf{w}^{\delta}(\cdot, t)\right\|_{L^{2}\left(\mathbb{T}^{N}\right)} \leq \frac{3 \widehat{C}_{1}}{2} \delta e^{\lambda_{\max } t}
$$

Furthermore, it follows from (109) and Lemma 4 that

$$
\begin{aligned}
\left\|\mathbf{w}^{\delta}(\cdot, t)\right\|_{H^{2}\left(\mathbb{T}^{N}\right)} & \leq \sqrt{\widehat{C}_{3}} \cdot \sqrt{\delta^{2}\left\|\mathbf{w}_{0}\right\|_{H^{2}\left(\mathbb{T}^{N}\right)}^{2}+\delta^{2} e^{2 \lambda_{\max } t}} \\
& \leq \sqrt{\widehat{C}_{3}} \cdot\left(\delta\left\|\mathbf{w}_{0}\right\|_{H^{2}\left(\mathbb{T}^{N}\right)}+\delta e^{\lambda_{\max } t}\right)
\end{aligned}
$$

for any $t \leq T^{* *}$.

Step 2. We establish $L^{2}$ estimate for $\mathbf{w}^{\delta}(x, t)$ of $0 \leq t \leq$ $\min \left\{T^{\delta}, T^{*}, T^{* *}\right\}$. Applying Duhamel's principle, we have

$$
\mathbf{w}^{\delta}(x, t)=\delta e^{L t} \mathbf{w}_{0}-\int_{0}^{t} e^{L(t-\tau)} N\left(\mathbf{w}^{\delta}(x, \tau)\right) d \tau
$$

where $N\left[\mathbf{w}^{\delta}(x, \tau)\right]=\left(N_{1}\left(\mathbf{w}^{\delta}(x, \tau)\right), N_{2}\left(\mathbf{w}^{\delta}(x, \tau)\right)\right), N_{1}\left(\mathbf{w}^{\delta}(x\right.$, $\tau)$ ), and $N_{2}\left(\mathbf{w}^{\delta}(x, \tau)\right)$ are given by (68) and (72). Using Lemma 1, it follows that

$$
\begin{aligned}
& \left\|\mathbf{w}^{\delta}(\cdot, t)-\delta e^{L t} \mathbf{w}_{0}\right\|_{L^{2}\left(\mathbb{T}^{N}\right)} \\
& =\left\|\int_{0}^{t} e^{L(t-\tau)} N\left(\mathbf{w}^{\delta}(x, \tau)\right) d \tau\right\|_{L^{2}\left(\mathbb{T}^{N}\right)} \\
& \leq \int_{0}^{t}\left\|e^{L(t-\tau)} N\left(\mathbf{w}^{\delta}(x, \tau)\right)\right\|_{L^{2}\left(\mathbb{T}^{N}\right)} d \tau \\
& \leq \widehat{C}_{1} \int_{0}^{t} e^{\lambda_{\max }(t-\tau)}\left\|N\left(\mathbf{w}^{\delta}(x, \tau)\right)\right\|_{L^{2}\left(\mathbb{T}^{N}\right)} d \tau, \\
& \left\|N\left(\mathbf{w}^{\delta}(x, \tau)\right)\right\|_{L^{2}\left(\mathbb{T}^{N}\right)} \\
& \leq\left\|N_{1}\left(\mathbf{w}^{\delta}(x, \tau)\right)\right\|_{L^{2}\left(\mathbb{T}^{N}\right)}+\left\|N_{2}\left(\mathbf{w}^{\delta}(x, \tau)\right)\right\|_{L^{2}\left(\mathbb{T}^{N}\right)} \\
& =\left\|-\left(u^{\delta}\right)^{2}-\beta u^{\delta} v^{\delta}\right\|_{L^{2}\left(\mathbb{T}^{N}\right)} \\
& +\| d_{2} d_{3} v^{\delta} \Delta u^{\delta}+2 d_{2} d_{3} \nabla u^{\delta} \nabla v^{\delta}+d_{2} d_{3} u^{\delta} \Delta v^{\delta} \\
& -\frac{\left(\mu u^{\delta}-v^{\delta}\right)^{2}}{m+u^{\delta}+\widetilde{u}} \|_{L^{2}\left(\mathbb{T}^{N}\right)} \\
& \leq(\beta+1)\left\|\mathbf{w}^{\delta}\right\|_{L^{\infty}\left(\mathbb{T}^{N}\right)}\left\|\mathbf{w}^{\delta}\right\|_{L^{2}\left(\mathbb{T}^{N}\right)}+2 d_{2} d_{3}\left\|\mathbf{w}^{\delta}\right\|_{L^{\infty}\left(\mathbb{T}^{N}\right)} \\
& \times\left\|\mathbf{w}^{\delta}\right\|_{H^{2}\left(\mathbb{T}^{N}\right)}+2 d_{2} d_{3}\left\|\nabla \mathbf{w}^{\delta}\right\|_{L^{4}\left(\mathbb{T}^{N}\right)}^{2} \\
& +\frac{(\mu+1)^{2}}{m}\left\|\mathbf{w}^{\delta}\right\|_{L^{\infty}\left(\mathbb{T}^{N}\right)}\left\|\mathbf{w}^{\delta}\right\|_{L^{2}\left(\mathbb{T}^{N}\right)} \\
& \leq \widehat{C}_{4}\left\|\mathbf{w}^{\delta}\right\|_{H^{2}\left(\mathbb{T}^{N}\right)}^{2},
\end{aligned}
$$

where $\widehat{C}_{4}=\beta+1+4 d_{2} d_{3}+(\mu+1)^{2} / m$. Therefore,

$$
\begin{aligned}
& \left\|\mathbf{w}^{\delta}(\cdot, t)-\delta e^{L t} \mathbf{w}_{0}\right\|_{L^{2}\left(\mathbb{T}^{N}\right)} \\
& \quad \leq \widehat{C}_{1} \widehat{C}_{4} \int_{0}^{t} e^{\lambda_{\max }(t-\tau)}\left\|\mathbf{w}^{\delta}(\tau)\right\|_{H^{2}\left(\mathbb{T}^{N}\right)}^{2} d \tau .
\end{aligned}
$$

Substituting (110) into (114), we obtain

$$
\begin{aligned}
& \left\|\mathbf{w}^{\delta}(\cdot, t)-\delta e^{L t} \mathbf{w}_{0}\right\|_{L^{2}\left(\mathbb{T}^{N}\right)} \\
& \leq \widehat{C}_{1} \widehat{C}_{3} \widehat{C}_{4} \int_{0}^{t} e^{\lambda_{\max }(t-\tau)} \\
& \quad \times\left(\delta^{2}\left\|\mathbf{w}_{0}\right\|_{H^{2}\left(\mathbb{T}^{N}\right)}^{2}+\delta^{2} e^{2 \lambda_{\max } \tau}\right) d \tau \\
& \leq \widehat{C}_{1} \widehat{C}_{3} \widehat{C}_{4}\left\{\frac{\delta\left\|\mathbf{w}_{0}\right\|_{H^{2}\left(\mathbb{T}^{N}\right)}^{2}}{\lambda_{\max }}+\frac{\delta e^{\lambda_{\max } t}}{\lambda_{\max }}\right\} \delta e^{\lambda_{\max } t} .
\end{aligned}
$$


Step 3. We prove by contradiction that

$$
T^{\delta}=\min \left\{T^{\delta}, T^{*}, T^{* *}\right\}
$$

for $\delta$ sufficiently small and

$$
\begin{gathered}
\theta<\min \left\{\frac{\lambda_{\max }}{4 \widehat{C}_{3} \widehat{C}_{4}}, \frac{\eta}{2 \sqrt{\widehat{C}_{3}}}, \frac{d_{1}}{8 \widehat{C}_{2} \sqrt{\widehat{C}_{3}}(1+\eta)^{2}},\right. \\
\left.\frac{A_{0} d_{2}\left(1+d_{3} \tilde{u}\right)}{4 \widehat{C}_{2} \sqrt{\widehat{C}_{3}}(1+\eta)^{2}}\right\} .
\end{gathered}
$$

If $T^{* *}=\min \left\{T^{\delta}, T^{*}, T^{* *}\right\}$, we can let $t=T^{* *} \leq T^{\delta}$ in (111) to obtain

$$
\begin{aligned}
\left\|\mathbf{w}^{\delta}\left(\cdot, T^{* *}\right)\right\|_{H^{2}\left(\mathbb{T}^{N}\right)} & \leq \sqrt{\widehat{C}_{3}}\left(\delta\left\|\mathbf{w}_{0}\right\|_{H^{2}\left(\mathbb{T}^{N}\right)}+\delta e^{\lambda_{\max } T^{* *}}\right) \\
& \leq \sqrt{\widehat{C_{3}}}\left(\delta\left\|\mathbf{w}_{0}\right\|_{H^{2}\left(\mathbb{T}^{N}\right)}+\theta\right) .
\end{aligned}
$$

By choosing $\delta$ sufficiently small, such that

$$
\begin{aligned}
& \sqrt{\widehat{C}_{3}} \delta\left\|\mathbf{w}_{0}\right\|_{H^{2}\left(\mathbb{T}^{N}\right)} \\
& \leq \frac{1}{2} \min \left\{\eta, \frac{d_{1}}{4 \widehat{C}_{2}(1+\eta)^{2}}, \frac{A_{0} d_{2}\left(1+d_{3} \tilde{u}\right)}{2 \widehat{C}_{2}(1+\eta)^{2}}\right\},
\end{aligned}
$$

and the choice of $\theta$ in (117), we have

$$
\begin{aligned}
& \left\|\mathbf{w}^{\delta}\left(\cdot, T^{* *}\right)\right\|_{H^{2}\left(\mathbb{T}^{N}\right)} \\
& \quad<\min \left\{\eta, \frac{d_{1}}{4 \widehat{C}_{2}(1+\eta)^{2}}, \frac{A_{0} d_{2}\left(1+d_{3} \tilde{u}\right)}{2 \widehat{C}_{2}(1+\eta)^{2}}\right\} .
\end{aligned}
$$

This is a contradiction to the definition of $T^{* *}$.

On the other hand, if $T^{*}=\min \left\{T^{\delta}, T^{*}, T^{* *}\right\}$, we can let $t=T^{*}$ in (115) to get

$$
\begin{aligned}
& \left\|\mathbf{w}^{\delta}\left(\cdot, T^{*}\right)-\delta e^{L T^{*}} \mathbf{w}_{0}\right\|_{L^{2}\left(\mathbb{T}^{N}\right)} \\
& \quad \leq \widehat{C}_{1} \widehat{C}_{3} \widehat{C}_{4}\left\{\frac{\delta\left\|\mathbf{w}_{0}\right\|_{H^{2}\left(\mathbb{T}^{N}\right)}^{2}}{\lambda_{\max }}+\frac{\theta}{\lambda_{\max }}\right\} \delta e^{\lambda_{\max } T^{*}} .
\end{aligned}
$$

By choosing $\delta$ sufficiently small, such that

$$
\widehat{C}_{3} \widehat{C}_{4} \frac{\delta\left\|\mathbf{w}_{0}\right\|_{H^{2}\left(\mathbb{T}^{N}\right)}^{2}}{\lambda_{\max }} \leq \frac{1}{4},
$$

and the choice of $\theta$ in (117), we have

$$
\left\|\mathbf{w}^{\delta}\left(\cdot, T^{*}\right)-\delta e^{L T^{*}} \mathbf{w}_{0}\right\|_{L^{2}\left(\mathbb{T}^{N}\right)}<\frac{\widehat{C}_{1}}{2} \delta e^{\lambda_{\max } T^{*}} .
$$

This again contradicts the definition of $T^{*}$. From these arguments, (116) holds.

Step 4. Rewriting the left-hand term in (115) as the form of (26), and separating $\mathbf{q} \in \Lambda_{\max }$ and moving $\mathbf{q} \notin \Lambda_{\max }$ to the right-hand side, it follows that

$$
\begin{aligned}
& \left\|\mathbf{w}^{\delta}(\cdot, t)-\delta e^{\lambda_{\max } t} \sum_{\mathbf{q} \in \Lambda_{\max }} w_{\mathbf{q}}^{+} \mathbf{r}_{+}(\mathbf{q}) e_{\mathbf{q}}(x)\right\|_{L^{2}\left(\mathbb{T}^{N}\right)} \\
& \leq \delta\left\|\sum_{\mathbf{q} \in \Lambda_{\max }} w_{\mathbf{q}}^{-} \mathbf{r}_{-}(\mathbf{q}) e^{\lambda_{\mathbf{q}}^{-} t} e_{\mathbf{q}}(x)\right\|_{L^{2}\left(\mathbb{T}^{N}\right)} \\
& +\delta \| \sum_{\mathbf{q} \in \Lambda_{1} \backslash \Lambda_{\max }}\left\{w_{\mathbf{q}}^{-} \mathbf{r}_{-}(\mathbf{q}) e^{\lambda_{\mathbf{q}}^{-} t}\right. \\
& \left.+w_{\mathbf{q}}^{+} \mathbf{r}_{+}(\mathbf{q}) e^{\lambda_{\mathbf{q}}^{+} t}\right\} e_{\mathbf{q}}(x) \|_{L^{2}\left(\mathbb{T}^{N}\right)} \\
& +\delta \| \sum_{\mathbf{q} \in \Lambda_{2}}\left\{w_{\mathbf{q}} \mathbf{r}(\mathbf{q})+w_{\mathbf{q}}^{\prime} \mathbf{r}^{\prime}(\mathbf{q})\right. \\
& \left.+w_{\mathbf{q}}^{\prime} \mathbf{r}(\mathbf{q}) t\right\} e^{\lambda_{\mathbf{q}} t} e_{\mathbf{q}}(x) \|_{L^{2}\left(\mathbb{T}^{N}\right)} \\
& +\delta \| \sum_{\mathbf{q} \in \Lambda_{3}}\left\{w _ { \mathbf { q } } ^ { \operatorname { R e } } \left(\operatorname{Re} \mathbf{r}(\mathbf{q}) \cos \left[\left(\operatorname{Im} \lambda_{\mathbf{q}}\right) t\right]\right.\right. \\
& \left.-\operatorname{Im} \mathbf{r}(\mathbf{q}) \sin \left[\left(\operatorname{Im} \lambda_{\mathbf{q}}\right) t\right]\right) \\
& +w_{\mathbf{q}}^{\operatorname{Im}}\left(\operatorname{Re} \mathbf{r}(\mathbf{q}) \sin \left[\left(\operatorname{Im} \lambda_{\mathbf{q}}\right) t\right]\right. \\
& \left.\left.+\operatorname{Im} \mathbf{r}(\mathbf{q}) \cos \left[\left(\operatorname{Im} \lambda_{\mathbf{q}}\right) t\right]\right)\right\} \\
& \times e^{\left(\operatorname{Re} \lambda_{\mathbf{q}}\right) t} e_{\mathbf{q}}(x) \|_{L^{2}\left(\mathbb{T}^{N}\right)} \\
& +\widehat{C}_{1} \widehat{C}_{3} \widehat{C}_{4}\left\{\frac{\delta\left\|\mathbf{w}_{0}\right\|_{H^{2}\left(\mathbb{T}^{N}\right)}^{2}}{\lambda_{\max }}+\frac{\delta e^{\lambda_{\max } t}}{\lambda_{\max }}\right\} \delta e^{\lambda_{\max } t} .
\end{aligned}
$$

Next, we process the first term on the right side of (124) to get

$$
\begin{gathered}
\delta\left\|\sum_{\mathbf{q} \in \Lambda_{\max }} w_{\mathbf{q}}^{-} \mathbf{r}_{-}(\mathbf{q}) e^{\lambda_{\mathbf{q}}^{-} t} e_{\mathbf{q}}(x)\right\|_{L^{2}\left(\mathbb{T}^{N}\right)} \\
=\delta\left(\sum_{\mathbf{q} \in \Lambda_{\max }}\left(w_{\mathbf{q}}^{-}\right)^{2}\left|\mathbf{r}_{-}(\mathbf{q})\right|^{2} e^{2 \lambda_{\mathbf{q}}^{-} t} d x\right)^{1 / 2} .
\end{gathered}
$$


Recall that there are only finite elements in $\Lambda_{\max }$ and for any $\mathbf{q} \in \Lambda_{\text {max }}$, there exists a constant $M_{0}$, such that $0<|\mathbf{q}|<M_{0}$. Therefore,

$$
\delta\left\|\sum_{\mathbf{q} \in \Lambda_{\max }} w_{\mathbf{q}}^{-} \mathbf{r}_{-}(\mathbf{q}) e^{\lambda_{\mathbf{q}}^{-} t} e_{\mathbf{q}}(x)\right\|_{L^{2}\left(\mathbb{T}^{N}\right)} \leq C \delta e^{\left(\lambda_{\max }-\sigma\right) t} .
$$

Similar to the arguments in the proof of Lemma 1, we can treat the second, third, and fourth terms to obtain some similar estimates as (126), and our theorem follows.

\section{Acknowledgments}

This work was supported by the National Natural Science Foundation of China as received Grant nos. 10871160 and 11361055, the Fundamental Research Funds for the Gansu University, and NWNU-LKQN-11-21 Foundations.

\section{References}

[1] A. M. Turing, "The chemical basis of morphogenesis," Philosophical Transactions of the Royal Society B, vol. 237, no. 641, pp. 37-72, 1952.

[2] T. K. Callahan and E. Knobloch, "Pattern formation in threedimensional reaction-diffusion systems," Physica D, vol. 132, no. 3, pp. 339-362, 1999.

[3] M. Wang, "Stability and Hopf bifurcation for a prey-predator model with prey-stage structure and diffusion," Mathematical Biosciences, vol. 212, no. 2, pp. 149-160, 2008.

[4] Y. Du and Y. Lou, "S-shaped global bifurcation curve and Hopf bifurcation of positive solutions to a predator-prey model," Journal of Differential Equations, vol. 144, no. 2, pp. 390-440, 1998.

[5] F. Yi, J. Wei, and J. Shi, "Bifurcation and spatiotemporal patterns in a homogeneous diffusive predator-prey system," Journal of Differential Equations, vol. 246, no. 5, pp. 1944-1977, 2009.

[6] Y. Du and S. B. Hsu, "A diffusive predator-prey model in heterogeneous environment," Journal of Differential Equations, vol. 203, no. 2, pp. 331-364, 2004.

[7] Y. Du and M. Wang, "Asymptotic behaviour of positive steady states to a predator-prey model," Proceedings of the Royal Society of Edinburgh A, vol. 136, no. 4, pp. 759-778, 2006.

[8] Y. Guo and W. A. Strauss, "Instability of periodic BGK equilibria," Communications on Pure and Applied Mathematics, vol. 48, no. 8, pp. 861-894, 1995.

[9] Y. Guo, "Instability of symmetric vortices with large charge and coupling constant," Communications on Pure and Applied Mathematics, vol. 49, no. 10, pp. 1051-1080, 1996.

[10] C. Bardos, Y. Guo, and W. Strauss, "Stable and unstable ideal plane flows," Chinese Annals of Mathematics B, vol. 23, no. 2, pp. 149-164, 2002.

[11] H. J. Hwang and Y. Guo, "On the dynamical Rayleigh-Taylor instability," Archive for Rational Mechanics and Analysis, vol. 167, no. 3, pp. 235-253, 2003.

[12] Y. Guo, C. Hallstrom, and D. Spirn, "Dynamics near an unstable Kirchhoff ellipse," Communications in Mathematical Physics, vol. 245, no. 2, pp. 297-354, 2004.

[13] Y. Guo and H. J. Hwang, "Pattern formation (I): the Keller-Segel model," Journal of Differential Equations, vol. 249, no. 7, pp. 15191530, 2010.
[14] Y. Guo and H. J. Hwang, "Pattern formation. II: the turing instability," Proceedings of the American Mathematical Society, vol. 135, no. 9, pp. 2855-2866, 2007.

[15] M. A. Aziz-Alaoui and M. Daher Okiye, "Boundedness and global stability for a predator-prey model with modified LeslieGower and Holling-type II schemes," Applied Mathematics Letters, vol. 16, no. 7, pp. 1069-1075, 2003.

[16] B. Dubey, B. Das, and J. Hussain, "A predator-prey interaction model with self and cross-diffusion," Ecological Modelling, vol. 141, no. 1-3, pp. 67-76, 2001.

[17] W. Wang, Y. Lin, L. Zhang, F. Rao, and Y. Tan, "Complex patterns in a predator-prey model with self and cross-diffusion," Communications in Nonlinear Science and Numerical Simulation, vol. 16, no. 4, pp. 2006-2015, 2011.

[18] Y. Du, R. Peng, and M. Wang, "Effect of a protection zone in the diffusive Leslie predator-prey model," Journal of Differential Equations, vol. 246, no. 10, pp. 3932-3956, 2009.

[19] R. Peng and M. Wang, "Positive steady states of the HollingTanner prey-predator model with diffusion," Proceedings of the Royal Society of Edinburgh A, vol. 135, no. 1, pp. 149-164, 2005.

[20] R. Peng, M. Wang, and G. Yang, "Stationary patterns of the Holling-Tanner prey-predator model with diffusion and crossdiffusion," Applied Mathematics and Computation, vol. 196, no. 2, pp. 570-577, 2008.

[21] O. A. Ladyženskaja, V. A. Solonnikov, and N. N. Uralcera, Linear and Quasilinear Equations of Parabolic Type, vol. 23 of Translations of Mathematical Monograph, AMS, Providence, RI, USA, 1968. 


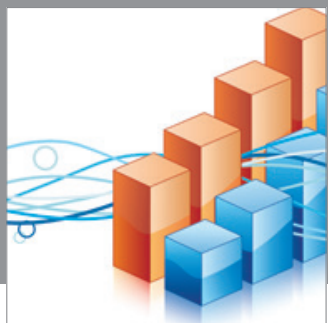

Advances in

Operations Research

mansans

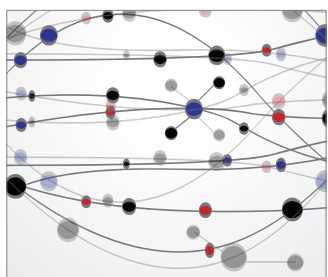

The Scientific World Journal
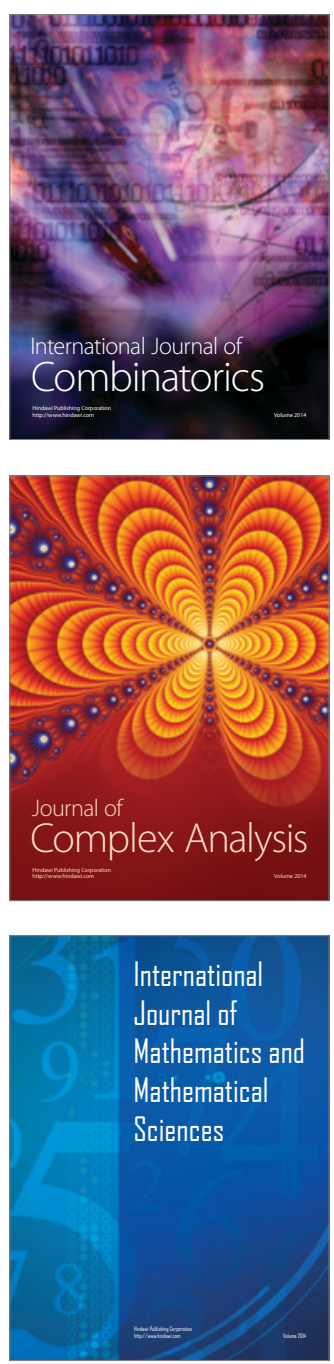
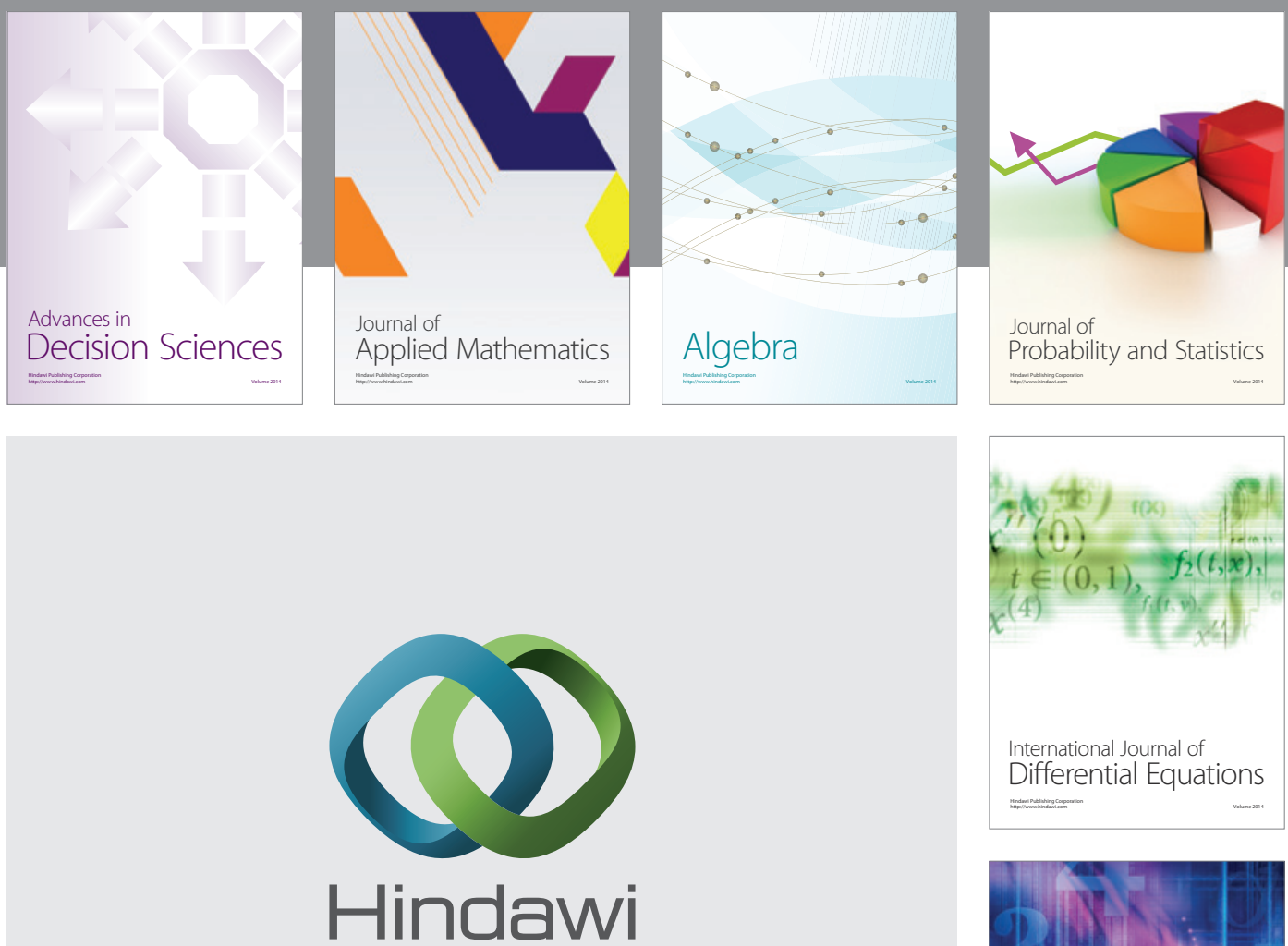

Submit your manuscripts at http://www.hindawi.com
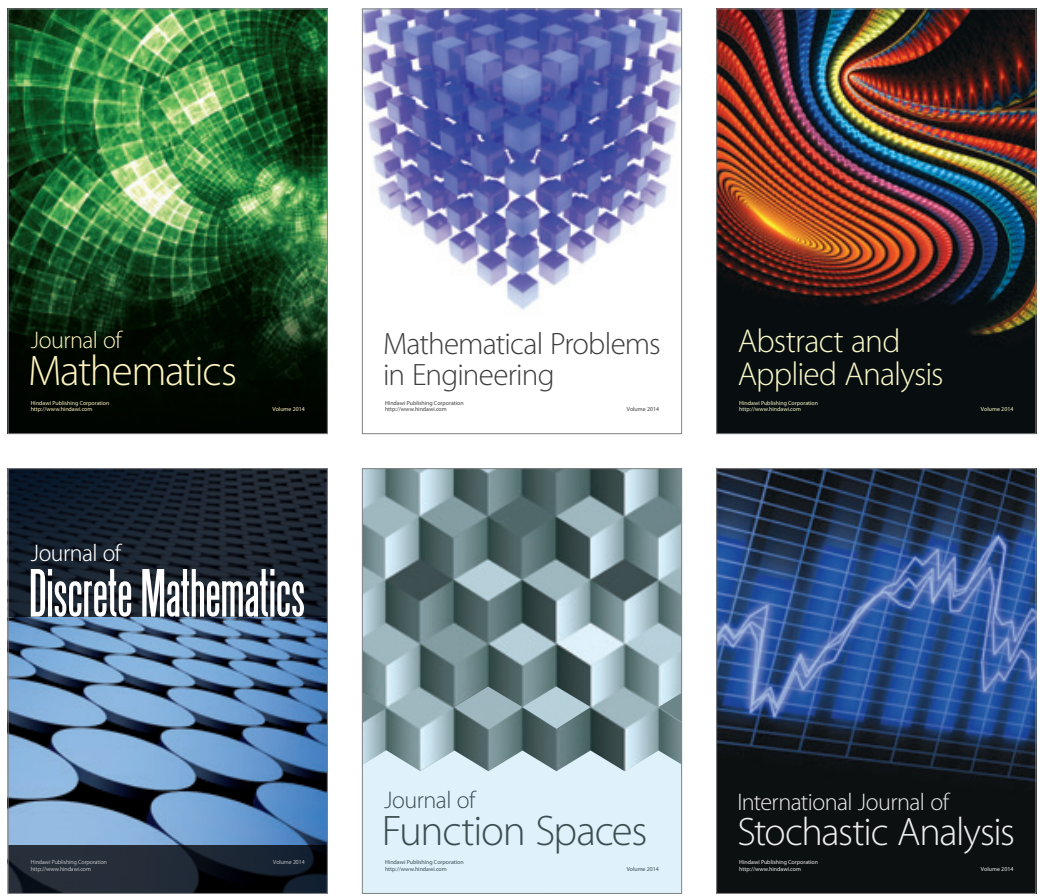

Journal of

Function Spaces

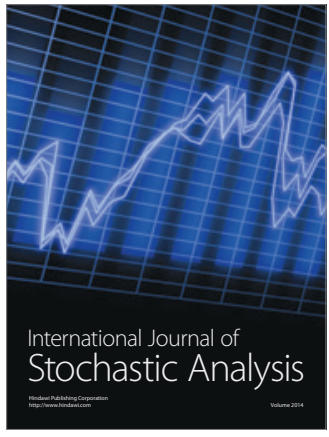

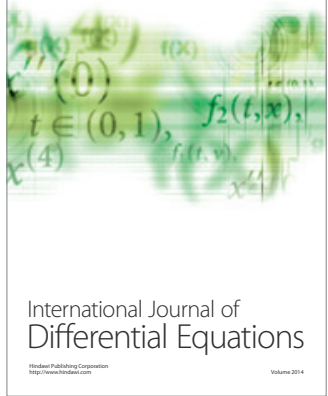
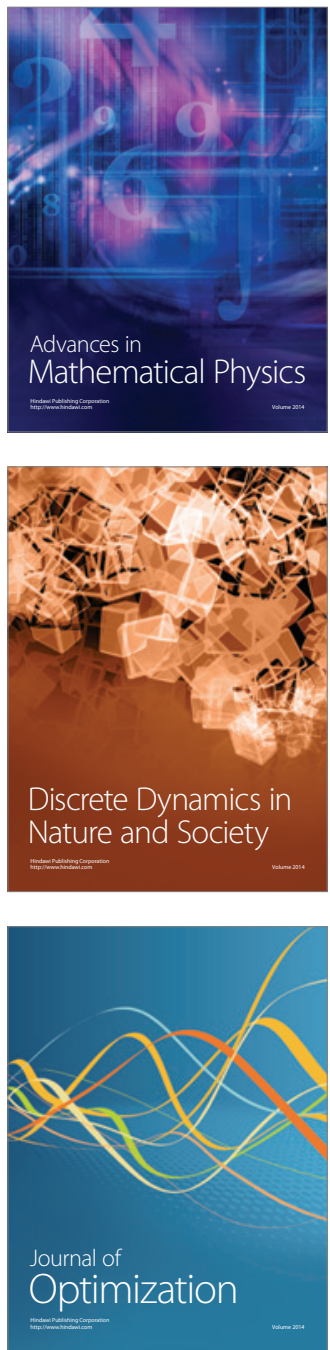